\title{
Synthesis of Heteroaryl ortho-Phenoxyethylamines via Suzuki Cross-Coupling: Easy Access to New Potential Scaffolds in Medicinal Chemistry
}

\author{
Leda Ivanova Manasieva \\ Battisti Umberto Maria \\ Adolfo Prandi \\ Livio Brasili \\ Silvia Franchini*
}

Dipartimento di Scienze della Vita, Università di Modena e Reggio Emilia, Via Campi 183, 41125 Modena, Italy silvia.franchini@unimore.it

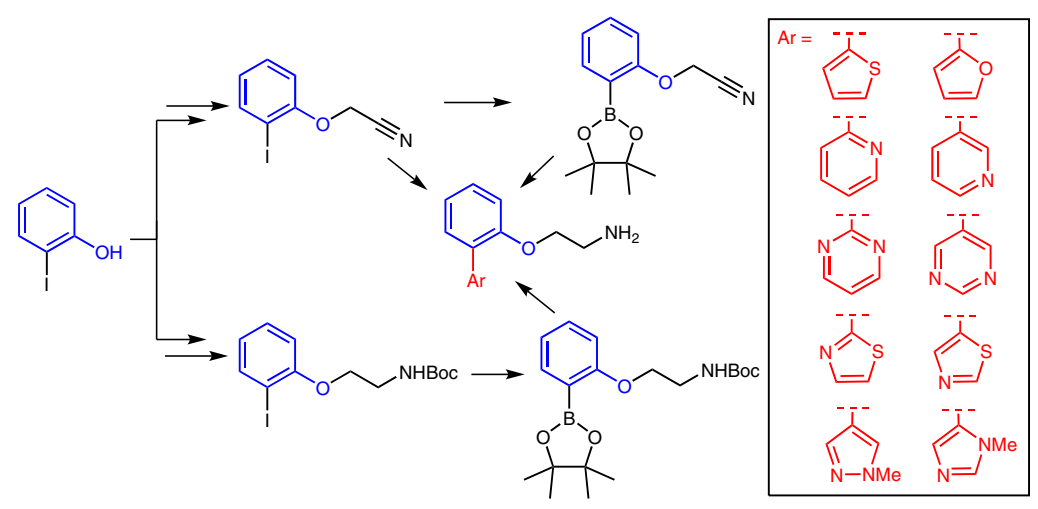

Received: 06.05.2015

Accepted after revision: 16.07.2015

Published online:

DOI: 10.1055/s-0035-1560456; Art ID: ss-2015-t0293-op

Abstract Heteroaryl ortho-phenoxyethylamines have been extensively employed in medicinal chemistry as privileged scaffolds for the design of highly potent and selective ligands. Herein an efficient, fast, and general method for the synthesis of heteroaryl phenoxyethylamines via Suzuki cross-coupling is reported. This approach offers the opportunity to obtain a large variety of biaryls incorporating five-membered (thiophene, furan, thiazole, pyrazole, imidazole) or six-membered (pyridine, pyrimidine) heteroaromatic rings for appropriate libraries of ligands. All the compounds presented here have never been synthesized before and a full structural characterization is given.

Key words C-C coupling, heterocycles, palladium, Suzuki-Miyaura cross-coupling, building blocks

Heteroaryl ortho-phenoxyethylamines have been extensively employed in medicinal chemistry as privileged scaffolds for the design of highly potent and selective ligands (Figure 1) for glycine transporter GlyT1 (1), ${ }^{1} \alpha_{1}$ adrenergic receptor $\mathbf{2},{ }^{2}$ DNA topoisomerases $3,{ }^{3}$ AMPA receptor $\mathbf{4},{ }^{4}$ and $5-\mathrm{HT}_{2 \mathrm{~A}}$ receptor 5.,6 Moreover, Trifenagrel (6), a potent inhibitor of arachidonate-induced platelets aggregation, ${ }^{7}$ moPhpC 7, fluorescent pyrrolocytosine nucleobases designed for tight binding to guanine, ${ }^{8}$ and some 2,3 - and 3,4diarylfurans, for example, $\mathbf{8}^{9}$ with anti-implantation activity, belong to this versatile class of heteroaryl ortho-phenoxyethylamines.

Despite the important role of these chemical entities in several fields of medicinal chemistry, a general method for heteroaryl ortho-phenoxyethylamine synthesis has not yet been reported. From a practical point of view, the assembly of these building blocks could be achieved via cross-coupling of two monocyclic units. Among the various method- ologies available, ${ }^{10}$ the Suzuki-Miyaura reaction, one of the most popular and powerful methods for the joining of arylaryl and aryl-heteroaryl moieties, was chosen. ${ }^{11}$ Thanks to its compatibility with a variety of functional groups, the stability, commercial availability, and low toxicity of a wide range of boronic starting materials, along with the ease of working up the reaction mixtures, this reaction has found many applications, in both research laboratories and largescale industrial processes. ${ }^{12}$

In this work, we describe the synthesis of several heteroaryl ortho-phenoxyethylamines via Suzuki cross-coupling. This approach offers the opportunity to obtain a large variety of biaryls incorporating five-membered electronrich (thiophene, furan), six-membered electron-poor (pyridine, pyrimidine), or five-membered rings with two heteroatoms (thiazole, pyrazole, imidazole) for appropriate libraries of ligands.

In an attempt to find a convenient and versatile strategy to build the ortho-substituted biaryl scaffold, we took into account several factors: $i$ ) the different nature of the heterocycle to be coupled: electron-rich or electron-poor; ii) the presence of sterically hindered ortho-substituted substrates; iii) the presence of $\mathrm{LiAlH}_{4}$-sensitive heterocycles such as pyrimidine; $i v$ ) the presence of heteroatoms such as sulfur or nitrogen that may drastically reduce the yields of some steps (i.e., reduction) due to the poisoning of the catalyst; and $v$ ) the opportunity to start from a common precursor (nitrile or masked amine) by inserting the structural diversity in the last step of the synthesis.

It is well known that the optimal conditions for Suzuki coupling is the electron-poor nature of aryl halide and the electron-rich nature of arylboronic acid. Keeping this in mind, we designed a synthetic approach, employing a common key intermediate $\mathbf{9}$, which directly underwent Suzuki 
<smiles>CN(CCOc1ccc(Br)cc1-c1cccs1)CC(=O)O</smiles><smiles>CN(C)C(=O)c1cccnc1N1CCC(NCCOc2ccccc2-c2cccnc2)CC1</smiles><smiles>[R]c1ccc2nc(-c3ccccc3OCCN([R])[R])[nH]c2c1</smiles><smiles>CN(C)CCOc1ccccc1-c1cnc(-c2ccccc2)[nH]1</smiles><smiles>C/C=C\CCCCCOc1ccc(NC(=O)c2cccc(OC)c2)cc1-c1ccnn1C</smiles><smiles>CN(C)CCOc1ccccc1-c1nc(-c2ccccc2)c(-c2ccccc2)[nH]1</smiles><smiles>CCn1cc2cc(-c3ccccc3OCCN)[nH]c2nc1=O</smiles><smiles>c1ccc(-c2cocc2-c2ccccc2OCCN2CCCC2)cc1</smiles>

Figure 1 Structures of heteroaryl ortho-phenoxyethylamine-based compounds

coupling in the case of electron-rich substrates or was converted into the corresponding pinacolboronic ester $\mathbf{1 0}$ and then coupled with the electron-poor aryl halide, as described in Scheme 1.

Alkylation of 2-iodophenol in the standard conditions reported for the Williamson reaction provided the aryl iodide 9 in high yield (89\%). Suzuki coupling of 2-(2-iodophe- noxy)acetonitrile (9) with an aryl pinacolboronic ester has recently been described..$^{13}$ However, while many references relating to the coupling of the $\boldsymbol{o}$-iodoanisole or higher homologues, such as $\mathbf{9}$, with a variety of boronic acids/esters have been reported, ${ }^{13,14}$ to the best of our knowledge, there are no examples of unsubstituted 0 -iodoanisole coupled with 2-thiophenyl- or 2-furanylboronic acids.

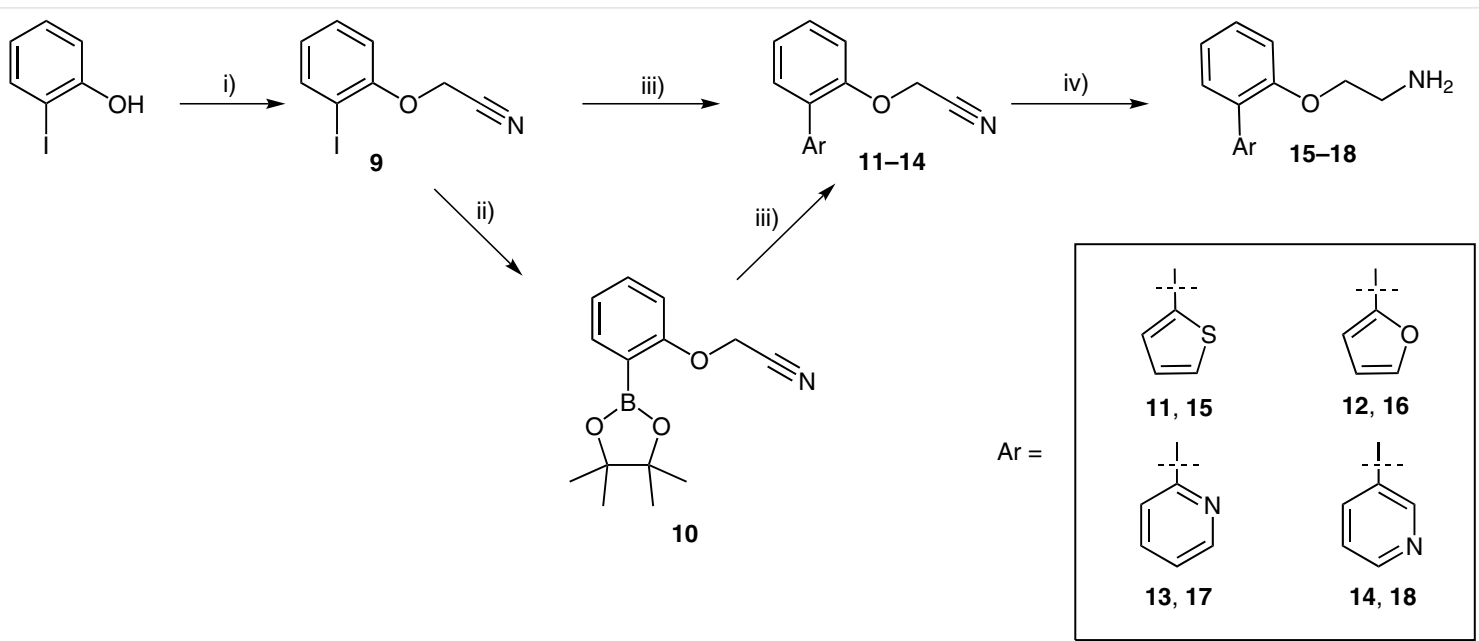

Scheme 1 Reagents and conditions: i) bromoacetonitrile, $\mathrm{K}_{2} \mathrm{CO}_{3}, 18$-crown- 6 ether, acetone, $55^{\circ} \mathrm{C}, 150 \mathrm{~W}, 1 \mathrm{~h}, 89 \%$; ii) see Table 1, entry 8, 95\%; iii) $\mathrm{ArB}(\mathrm{OH})_{2}$ (for 11, 12) or $\mathrm{ArBr}$ (for 13, 14), $\mathrm{Na}_{2} \mathrm{CO}_{3}, \mathrm{Pd}\left(\mathrm{PPh}_{3}\right)_{4}$, toluene-EtOH (3:1 v/v), $90{ }^{\circ} \mathrm{C}, 8 \mathrm{~h}, 70 \%(11), 93 \%$ (12), 68\% (13), 40\% (14); iv) LiAlH $\mathrm{Et}_{2} \mathrm{O}, 0$ to $25^{\circ} \mathrm{C}, 1 \mathrm{~h}, 92 \%$ (15), 60\% (16), $79 \%$ (17), $79 \%$ (18). 
In this paper, we describe the synthesis of exemplary 2arylfuran or -thiophene derivatives, via palladium-catalyzed coupling, starting from the corresponding electronically activated boronic acids. It is known that 2-thiophenylor 2-furanylboronic acids easily undergo deboronation under the basic conditions of the Suzuki-Miyaura coupling. ${ }^{15}$ Here we have developed an optimized procedure in which the degree of deboronation is negligible or absent, as attested by the good/excellent yields reported for heterobiaryls 11 and 12 (70 and 93\%, respectively).

We found that the best reaction conditions were tetrakis(triphenylphosphine)palladium( $(0)$ as a catalyst in combination with the cheap base potassium carbonate; a mixture of toluene and ethanol was more effective than DMF as a reaction solvent, as attested by the higher yields, compared to those previously reported. ${ }^{13}$ However, when a mixture of toluene-ethanol $(1: 1 \mathrm{v} / \mathrm{v})$ was employed, along with the desired product 11 (55\% yield), the corresponding ethyl ester (14\%) was isolated, due to the alcoholysis of the nitrile. Since the presence of an alcohol may improve water/toluene miscibility and consequently the activation of the boronic acid to boronate by the hydroxyl anion, in order to prevent the formation of the ethyl ester, the amount of ethanol was lowered (toluene-ethanol, 3:1 v/v). Under these conditions the ester was found in trace amounts and $\mathbf{1 1}$ was isolated in good yield (70\%). By using the same coupling conditions described for 11, the 2-furanyl derivative 12 was synthetized in excellent yield (93\%) without any optimization steps.

These coupling conditions allow a variety of heterobiaryls to be obtained in good/high yields, starting from the corresponding electron-rich boronic acids, even in the presence of a base sensitive group.

Conversely, since it is known that Suzuki reactions of electron-deficient 2-heterocyclic boronates, such as 2-pyridyl, generally give low conversions, ${ }^{16} \mathbf{1 3}$ was obtained by reacting the corresponding commercially available bromo derivative with the pinacolboronic ester 10. Pinacol boronates are a very attractive synthon since they are air-, moisture-, temperature-, and chromatography-stable. However, for ortho-substituted substrates, as for $\mathbf{9}$, the synthesis of the corresponding boronic ester provides very low yields and also requires harsh conditions, due to the steric hindrance created by the ortho-substituent. ${ }^{17}$ In an attempt to obtain the pinacolboronic intermediate $\mathbf{1 0}$ in high yields, the results published by several groups concerning the Suzuki coupling of sterically hindered substrates were investigated. ${ }^{17,18}$ In fact, it has been reported that the employment of a phosphine ligand might improve the borylation of ortho-substituted aryl halides under mild conditions.

A series of commercially available palladium-based catalysts such as tetrakis(triphenylphosphine)palladium(0) $\left[\mathrm{Pd}\left(\mathrm{PPh}_{3}\right)_{4}\right], \quad$ tris(dibenzylideneacetone)dipalladium $(0)$ $\left[\mathrm{Pd}_{2}(\mathrm{dba})_{3}\right]$, and palladium(II) acetate $\left[\mathrm{Pd}(\mathrm{OAc})_{2}\right]$ alone or in combination with palladium ligands such as JohnPhos [1,1'biphenyl]-2-yl-di-tert-butylphosphine or Cy-JohnPhos [(2biphenyl)dicyclohexylphosphine], were considered.

In Table 1, the optimization of the borylation reaction, including solvent $\left(\mathrm{Et}_{3} \mathrm{~N}\right.$ or $\left.\mathrm{DMF}\right)$ and reaction time, is shown. The optimal reaction conditions were found to be associated with the employment of $\mathrm{Pd}(\mathrm{OAc})_{2}$ and a biphenylphosphine ligand. In particular, a molar ratio of 4:1 ligand (Cy-JohnPhos) to catalyst $\left[\mathrm{Pd}(\mathrm{OAc})_{2}\right]$ afforded $\mathbf{1 0}$ with the highest yield (95\%) and the lowest reaction time (2 h) (Table 1 , entry 8$)$.

Table 1 Optimization of the Borylation Reaction of 2-(2-lodophenoxy)acetonitrile (9)
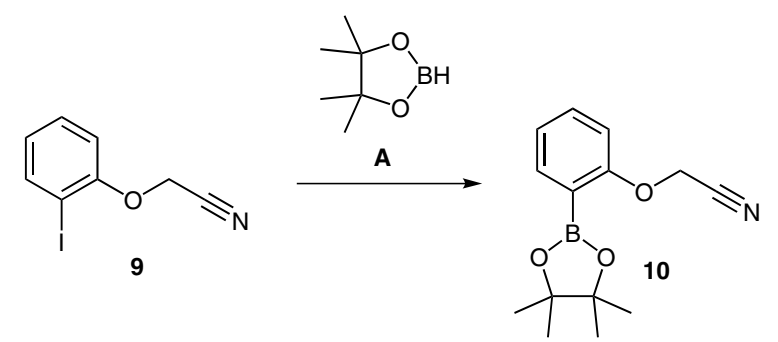<smiles>CC(C)(C)P(c1ccccc1-c1ccccc1)C(C)(C)C</smiles>

JohnPhos

(B)

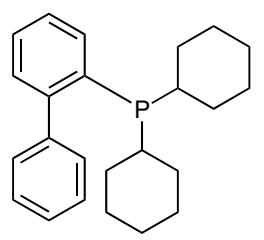

Cy-JohnPhos

(C)

\begin{tabular}{|c|c|c|c|c|}
\hline Entry & Pd catalyst & $\begin{array}{l}\text { Temp } \\
\left({ }^{\circ} \mathrm{C}\right)\end{array}$ & $\begin{array}{l}\text { Time } \\
\text { (h) }\end{array}$ & $\begin{array}{l}10 \text { Yield } \\
(\%)^{b}\end{array}$ \\
\hline 1 & $5 \mathrm{~mol} \% \mathrm{Pd}\left(\mathrm{PPh}_{3}\right)_{4}$ & 100 & 10 & 0 \\
\hline 2 & $10 \mathrm{~mol} \% \mathrm{Pd}\left(\mathrm{PPh}_{3}\right)_{4}$ & 100 & 10 & 0 \\
\hline 3 & $5 \mathrm{~mol}^{2} \mathrm{Pd}_{2} \mathrm{dba}_{3}$ & 100 & 10 & 0 \\
\hline 4 & $10 \mathrm{~mol}_{\%} \mathrm{Pd}_{2} \mathrm{dba}_{3}$ & 100 & 10 & 0 \\
\hline 5 & $5 \mathrm{~mol} \% \mathrm{Pd}(\mathrm{OAc})_{2}, 10 \mathrm{~mol} \% \mathbf{B}$ & 100 & 20 & 33 \\
\hline 6 & $5 \mathrm{~mol} \% \mathrm{Pd}(\mathrm{OAc})_{2}, 20 \mathrm{~mol} \% \mathbf{B}$ & 100 & 20 & 54 \\
\hline 7 & $5 \mathrm{~mol} \% \mathrm{Pd}(\mathrm{OAc})_{2}, 10 \mathrm{~mol} \% \mathrm{C}$ & 100 & 5 & 82 \\
\hline 8 & $5 \mathrm{~mol} \% \mathrm{Pd}(\mathrm{OAc})_{2}, 20 \mathrm{~mol} \% \mathrm{C}$ & 100 & 2 & 95 \\
\hline
\end{tabular}

Thus, the pinacolboronic ester $\mathbf{1 0}$ was allowed to react with 2-bromo- or 3-bromopyridine using the same coupling conditions described for $\mathbf{1 1}$, to afford the pyridinyl derivatives 13 and 14 in good (68\%) and moderate (40\%) yields, respectively. The same conditions were successfully applied for the coupling of highly electronically deactivated aryl halides, such as bromopyrimidine (data not shown). 
<smiles>CC(C)(C)OC(=O)NCCCl</smiles>

19

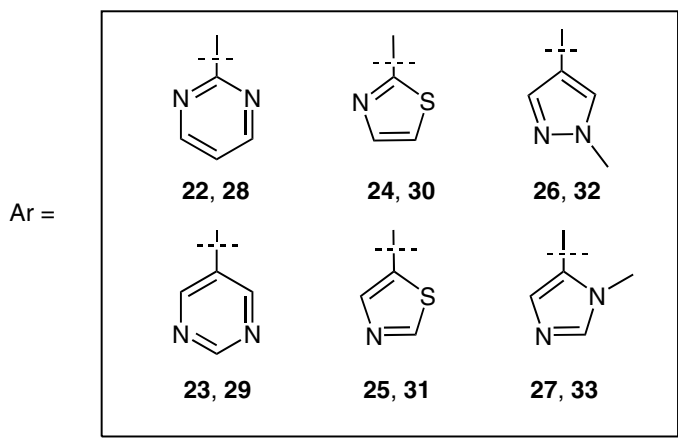<smiles>CC(C)(C)OC(=O)NCCOc1ccccc1I</smiles>

20
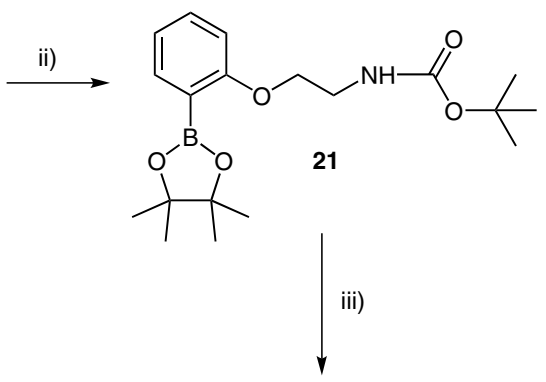<smiles>NCCOc1ccccc1Br</smiles>

28-33

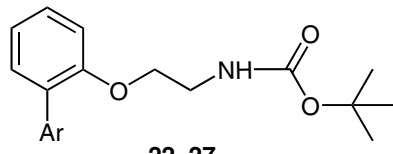

22-27

Scheme 2 Reagents and conditions: i) $\mathrm{K}_{2} \mathrm{CO}_{3}, \mathrm{Kl}, \mathrm{DMF}, 160^{\circ} \mathrm{C}, 4 \mathrm{~h}, 95 \%$; ii) $\mathrm{Et}_{3} \mathrm{~N}$, pinacolborane, $\mathrm{Pd}(\mathrm{OAc})_{2}$, (2-biphenyl)dicyclohexylphosphine, 1,4dioxane, $100{ }^{\circ} \mathrm{C}, 2 \mathrm{~h}, 99 \%$; iii) $\mathrm{ArBr}, \mathrm{Na}_{2} \mathrm{CO}_{3}, \mathrm{Pd}\left(\mathrm{PPh}_{3}\right)$, toluene-EtOH (3:1, v/v), $90{ }^{\circ} \mathrm{C}, 8 \mathrm{~h}, 34 \%$ (22), $42 \%$ (23), 88\% (24), 33\% (25), 54\% (25), 76\% (27); iv) $\mathrm{HCl}$, EtOH, 0 to $70{ }^{\circ} \mathrm{C}, 2$ h, 82\% (28), 72\% (29), 77\% (30), 78\% (31), 83\% (32), 99\% (33).

Finally, the desired amines $\mathbf{1 5 - 1 8}$ were obtained in good/high yields (60-92\%) after reduction of the nitriles 11-14 by $\mathrm{LiAlH}_{4}$. Unfortunately, in the case of pyrimidinyl biaryls the use of highly reactive reducing agents, such as $\mathrm{LiAlH}_{4}$, resulted in the degradation of the pyridimidine moiety.

Investigation with thiazole analogues led to similar results. This side-reaction can be explained by the low aromatic stability and/or the electron-poor nature of these rings that easily undergo reduction under the conditions required for the conversion of the nitrile to amine. An attempt to increase the chemoselectivity (i.e., by lowering the temperature to $0{ }^{\circ} \mathrm{C}$ ) resulted in very low yields of the desired amine.

Thus, for the synthesis of biaryls incorporating $\mathrm{LiAlH}_{4}{ }^{-}$ sensitive heterocycles, such as pyrimidine, thiazole, or pyrazole, a second version of the synthetic pathway was designed that involved the introduction of a masked amino group before the construction of the biaryl scaffold. In particular, the tert-butyloxycarbonyl group (Boc) was chosen as the protecting group for the primary amine, due to its stability under the basic conditions required for the coupling and to the ease of removal (Scheme 2). Briefly, the Boc-protected amine 19, synthesized in accordance with the literature, ${ }^{19}$ was reacted with 2 -iodophenol to obtain the intermediate $\mathbf{2 0}$ that was quantitatively converted into the corresponding pinacolboronate derivative $\mathbf{2 1}$, using the optimized conditions reported for $\mathbf{1 0}$ (Table 1, entry 8). The pinacolboronic ester 21 was reacted with the selected commercially available bromo derivatives under the same con- ditions as described for $\mathbf{1 1}$ to provide the biaryls $\mathbf{2 2 - 2 7}$. Finally, deprotection of 22-27 under acidic conditions afforded the desired amines 28-33.

In summary, a versatile and efficient method for the synthesis of heteroaryl ortho-phenoxyethylamines has been described. This approach offers the opportunity to obtain a large variety of biaryls incorporating five-membered rings with one (thiophene, furan) or two (thiazole, pyrazole, imidazole) heteroatoms and six-membered (pyridine, pyrimidine) heteroaromatic cycles starting from electronpoor aryl halide or electron-rich arylboronic acids. To date, a number of new compounds have been synthesized using this procedure and currently they are under investigation in a field as important as medicinal chemistry.

Reagents, solvents, and other chemicals were used as purchased without further purification, unless otherwise specified. Air- or moisture-sensitive reactants and solvents were employed in reactions carried out under $\mathrm{N}_{2}$ atmosphere, unless otherwise noted. Flash column chromatography purification (medium-pressure liquid chromatography) was carried out using Merck silica gel 60 (230-400 mesh, ASTM). The structures of all the isolated compounds were ensured by NMR spectra and elemental analysis $(\mathrm{C}, \mathrm{H}, \mathrm{N})$. NMR data $\left({ }^{1} \mathrm{H}\right.$ and ${ }^{13} \mathrm{C}$, 1D and 2D experiments) were obtained using a DPX 400 Avance spectrometer (Bruker). Chemical shifts are expressed in $\delta$ (ppm). ${ }^{1} \mathrm{H}$ NMR chemical shifts are relative to TMS as an internal standard. ${ }^{13} \mathrm{C}$ NMR chemical shifts are relative to internal TMS at $\delta=0.0$ or to the ${ }^{13} \mathrm{C}$ signal of solvent: $\mathrm{CDCl}_{3} \delta=77.04, \mathrm{CD}_{3} \mathrm{OD} \delta=49.8$, DMSO- $d_{6} \delta=39.5 .{ }^{1} \mathrm{H}$ NMR data are reported as follows: chemical shift, multiplicity (standard abbreviations), coupling constants, number of protons, and assignment. The elemental analysis was performed on a Carlo Erba 1106 Analyzer in the Microanalysis Laboratory of the Life Sciences Department of the Università degli Studi di Modena e Reggio Emilia and the results shown here are within $\pm 0.4 \%$ of the theoretical values. 


\section{2-(2-Iodophenoxy)acetonitrile (9)}

A round-bottomed flask was charged with 0 -iodophenol (2 g, 9.09 mmol), bromoacetonitrile (3.16 g, $26.0 \mathrm{mmol}), \mathrm{K}_{2} \mathrm{CO}_{3}(6.28 \mathrm{~g}, 45.0$ $\mathrm{mmol})$, and a catalytic amount of 18 -crown-6. The reaction mixture was suspended in anhydrous acetone ( $35 \mathrm{~mL}$ ) and heated in 'openvessel' conditions under microwave irradiation at $55^{\circ} \mathrm{C}, 150 \mathrm{~W}$ for 1 h. Then, the reaction mixture was cooled to r.t. and the solvent was evaporated under reduced pressure. The crude product was purified using flash column chromatography to provide the title compound as a colorless oil; yield: $2.114 \mathrm{~g}(8.16 \mathrm{mmol}, 89 \%)$.

IR (neat): 1493, 1238, 1098, 1031, $780 \mathrm{~cm}^{-1}$.

${ }^{1} \mathrm{H}$ NMR (400 MHz, $\mathrm{CDCl}_{3}$ ): $\delta=4.75\left(\mathrm{~s}, 2 \mathrm{H}, \mathrm{CH}_{2}\right.$ ), 6.80 (dt, $J=1.2,7.7$ $\mathrm{Hz}, 1 \mathrm{H}, \mathrm{CH}-4 \mathrm{Ar}$ ), 6.92 (dd, J=1.2, $7.7 \mathrm{~Hz}, 1 \mathrm{H}, \mathrm{CH}-6 \mathrm{Ar}$ ), 7.30 (dt, $J=1.5,7.7 \mathrm{~Hz}, 1 \mathrm{H}, \mathrm{CH}-5 \mathrm{Ar}$ ), 7.75 (dd, $J=1.5,7.7 \mathrm{~Hz}, 1 \mathrm{H}, \mathrm{CH}-3 \mathrm{Ar}$ ).

${ }^{13} \mathrm{C}$ NMR $\left(100 \mathrm{MHz}, \mathrm{CDCl}_{3}\right): \delta=54.9\left(\mathrm{CH}_{2}\right), 86.7(\mathrm{C}-2 \mathrm{Ar}), 113.7(\mathrm{C}-6$ Ar), 1146 (CN), 125.2 (C-4 Ar), 129.8 (C-5 Ar), 140.2 (C-3 Ar), 155.5 (C-1 Ar).

Anal. Calcd for $\mathrm{C}_{8} \mathrm{H}_{6} \mathrm{INO}$ : C, 37.09; H, 2.33; N, 5.41. Found: C, 37.24; $\mathrm{H}$, $2.46 ; \mathrm{N}, 5.56$.

\section{2-[2-(4,4,5,5-Tetramethyl-1,3-dioxolan-2-yl)phenoxy]acetonitrile (10)}

Pinacolborane (4.93 mL, $34.0 \mathrm{mmol})$ and $\mathrm{Et}_{3} \mathrm{~N}(7.28 \mathrm{~mL}, 52.4 \mathrm{mmol})$ were added to a solution of $\mathbf{9}(3.4 \mathrm{~g}, 13.1 \mathrm{mmol})$ in anhydrous 1,4dioxane ( $35 \mathrm{~mL}$ ) under $\mathrm{N}_{2}$. The mixture was degassed, purged with $\mathrm{N}_{2}$ for 10 minutes, and $\mathrm{Pd}(\mathrm{OAc})_{2}(0.146 \mathrm{~g}, 0.65 \mathrm{mmol}, 5 \mathrm{~mol} \%)$ and (2biphenyl)dicyclohexylphosphine (0.92 g, $2.62 \mathrm{mmol}, 20 \mathrm{~mol} \%$ ) were added. The mixture was vigorously stirred at $100{ }^{\circ} \mathrm{C}$ under $\mathrm{N}_{2}$ for 90 $\mathrm{min}$. Then, the reaction mixture was allowed to cool to r.t. and carefully quenched with sat. aq $\mathrm{NH}_{4} \mathrm{Cl}$ at $0{ }^{\circ} \mathrm{C}$. The mixture was then extracted with $\mathrm{CH}_{2} \mathrm{Cl}_{2}$ and the combined organic layers were dried $\left(\mathrm{Na}_{2} \mathrm{SO}_{4}\right)$, filtered, and evaporated. The crude product was purified using flash column chromatography to give the title compound as a colorless oil; yield: $3.22 \mathrm{~g}$ (12.4 mmol, 95\%).

IR (neat): 3290 (br), 1774, 1511, 1438, 1366, 1309, $1108 \mathrm{~cm}^{-1}$.

${ }^{1} \mathrm{H} \mathrm{NMR}\left(400 \mathrm{MHz}, \mathrm{CDCl}_{3}\right): \delta=1.37\left[\mathrm{~s}, 12 \mathrm{H}, 2 \times \mathrm{C}\left(\mathrm{CH}_{3}\right)_{2}\right], 4.79(\mathrm{~s}, 2 \mathrm{H}$, $\left.\mathrm{CH}_{2}\right), 7.01(\mathrm{~d}, J=7.5 \mathrm{~Hz}, 1 \mathrm{H}, \mathrm{CH}-6 \mathrm{Ar}), 7.13(\mathrm{t}, J=7.5 \mathrm{~Hz}, 1 \mathrm{H}, \mathrm{CH}-4 \mathrm{Ar})$, 7.46 (t, J = 7.5 Hz, $1 \mathrm{H}, \mathrm{CH}-5 \mathrm{Ar}$ ), 7.79 (d, J= 7.5 Hz, $1 \mathrm{H}, \mathrm{CH}-3 \mathrm{Ar}$ ).

${ }^{13} \mathrm{C} \mathrm{NMR}\left(100 \mathrm{MHz}, \mathrm{CDCl}_{3}\right): \delta=24.8\left[2 \times \mathrm{C}\left(\mathrm{CH}_{3}\right)_{2}\right], 56.0\left(\mathrm{CH}_{2}\right), 83.8[2 \times$ $\mathrm{C}\left(\mathrm{CH}_{3}\right)_{2}$ ], $1149(\mathrm{CN}), 115.3$ (C-6 Ar), $115.6(\mathrm{C}-2 \mathrm{Ar}), 123.9$ (C-4 Ar), 132.8 (C-5 Ar), 137.3 (C-3 Ar), 161.6 (C-1 Ar).

Anal. Calcd for $\mathrm{C}_{14} \mathrm{H}_{18} \mathrm{BNO}_{3}$ : C, 64.90; $\mathrm{H}, 7.00 ; \mathrm{N}, 5.41$. Found: C, 65.11; $\mathrm{H}, 7.06 ; \mathrm{N}, 5.38$.

\section{Suzuki-Coupling Reactions of 9; General Procedure A}

A $100 \mathrm{~mL}$ round-bottomed flask, equipped with a condenser and a magnetic stir bar, was charged with 0 -iodophenoxyacetonitrile $(\mathbf{9} ; 1$ equiv), $\mathrm{Na}_{2} \mathrm{CO}_{3}$ (2 equiv), and toluene-EtOH mixture (20 mL, 3:1 v/v). Thereafter, the corresponding commercially available arylboronic acid (1.2 equiv) was added to the resulting suspension. The mixture was degassed, purged with $\mathrm{N}_{2}$ for $15-20 \mathrm{~min}$, and then $\mathrm{Pd}\left(\mathrm{PPh}_{3}\right)_{4}(10$ mol\%) was added. The reaction mixture was heated at $90{ }^{\circ} \mathrm{C}$ for $8 \mathrm{~h}$ under stirring. The reaction mixture was allowed to cool to r.t., brine $(20 \mathrm{~mL})$ was added, and the mixture stirred for $30 \mathrm{~min}$. The organic layer was then diluted with EtOAc, transferred to a separatory funnel, washed with $\mathrm{H}_{2} \mathrm{O}$ and brine, dried $\left(\mathrm{Na}_{2} \mathrm{SO}_{4}\right)$, filtered, and concentrated under reduced pressure. The resulting residue was purified on silica gel to provide the title compound as an oil.

\section{2-[2-(Thiophen-2-yl)phenoxy]acetonitrile (11)}

The title compound was obtained by following General Procedure A from $9(0.400 \mathrm{~g}, 1.54 \mathrm{mmol})$ and 2-thiophenylboronic acid $(0.237 \mathrm{~g}$, $1.85 \mathrm{mmol})$ as a dark oil; yield: $0.233 \mathrm{~g}(1.08 \mathrm{mmol}, 70 \%)$.

IR (neat): 3392, 1662, 1554, 1401, 1368, 1345, 1121, 892, $840 \mathrm{~cm}^{-1}$.

${ }^{1} \mathrm{H}$ NMR (400 MHz, $\left.\mathrm{CDCl}_{3}\right): \delta=4.72\left(\mathrm{~s}, 2 \mathrm{H}, \mathrm{CH}_{2}\right), 6.98-7.06(\mathrm{~m}, 2 \mathrm{H}$, CH-6 Ar, CH-4 Thio), 7.09 (dt, J=1.2, 7.6 Hz, 1 H, CH-4 Ar), 7.24 (dt, $J=1.6,7.6 \mathrm{~Hz}, 1 \mathrm{H}, \mathrm{CH}-5 \mathrm{Ar}$ ), 7.29 (dd, $J=1.1,5.1 \mathrm{~Hz}, 1 \mathrm{H}, \mathrm{CH}-3 \mathrm{Thio}$ ), 7.37 (dd, $J=1.1,3.7 \mathrm{~Hz}, 1 \mathrm{H}, \mathrm{CH}-5$ Thio), 7.58 (dd, $J=1.6,7.6 \mathrm{~Hz}, 1 \mathrm{H}$, $\mathrm{CH}-3 \mathrm{Ar})$.

${ }^{13} \mathrm{C}$ NMR (100 MHz, $\left.\mathrm{CDCl}_{3}\right): \delta=54.8\left(\mathrm{CH}_{2}\right), 114.4(\mathrm{C}-6 \mathrm{Ar}), 1149(\mathrm{CN})$, 123.9 (C-4 Ar), 124.9 (C-2 Ar), 126.1 (C-3 Thio), 126.3 (C-5 Thio), 127.1 (C-4 Thio), 128.6 (C-5 Ar), 129.7 (C-3 Ar), 138.1 (C-1 Thio), 152.7 (C-1 Ar).

Anal. Calcd for $\mathrm{C}_{12} \mathrm{H}_{9} \mathrm{NOS}$ : C, 66.95; $\mathrm{H}, 4.21$; N, 6.51. Found: C, 66.99; $\mathrm{H}, 4.23 ; \mathrm{N}, 6.67$.

\section{2-[2-(Furan-2-yl)phenoxy]acetonitrile (12)}

The title compound was obtained by following General Procedure A from 9 (0.250 g, $0.97 \mathrm{mmol}$ ) and 2-furanylboronic acid (0.431 g, 3.86 $\mathrm{mmol})$ as a yellow oil; yield: $0.177 \mathrm{~g}(0.89 \mathrm{mmol}, 93 \%)$.

IR (neat): 3396, 3100, 1666, 1554, 1352, 1107, 950, $811 \mathrm{~cm}^{-1}$.

${ }^{1} \mathrm{H} \mathrm{NMR}\left(400 \mathrm{MHz}, \mathrm{CDCl}_{3}\right): \delta=4.85\left(\mathrm{~s}, 2 \mathrm{H}, \mathrm{CH}_{2}\right), 6.52(\mathrm{dd}, J=1.7,3.4$ $\mathrm{Hz}, 1 \mathrm{H}, \mathrm{CH}-4$ Fur), 6.90 (dd, J= 0.7, $3.4 \mathrm{~Hz}, 1 \mathrm{H}, \mathrm{CH}-3 \mathrm{Fur}$ ), 7.04 (dd, $J=1.3,7.7 \mathrm{~Hz}, 1 \mathrm{H}, \mathrm{CH}-6 \mathrm{Ar}), 7.18(\mathrm{dt}, J=1.3,7.7 \mathrm{~Hz}, 1 \mathrm{H}, \mathrm{CH}-4 \mathrm{Ar})$, 7.29 (dt, $J=1.7,7.7 \mathrm{~Hz}, 1 \mathrm{H}, \mathrm{CH}-5 \mathrm{Ar}$ ), 7.50 (dd, $J=0.7,1.7 \mathrm{~Hz}, 1 \mathrm{H}, \mathrm{CH}-$ 5 Fur), 7.90 (dd, $J=1.7,7.7 \mathrm{~Hz}, 1 \mathrm{H}, \mathrm{CH}-3 \mathrm{Ar}$ ).

${ }^{13} \mathrm{C}$ NMR (100 MHz, $\left.\mathrm{CDCl}_{3}\right): \delta=54.9\left(\mathrm{CH}_{2}\right), 109.8$ (C-5 Fur), $111.8(\mathrm{C}-4$ Fur), 114.5 (C-6 Ar), 1148 (CN), 123.7 (C-4 Ar), 125.3 (C-2 Ar), 128.6 (C-5 Ar), 129.7 (C-3 Ar), 141.1 (C-3 Fur), 150.3 (C-1 Fur), 152.8 (C-1 Ar).

Anal. Calcd for $\mathrm{C}_{12} \mathrm{H}_{9} \mathrm{NO}_{2}$ : C, 72.35; H, 4.55; N, 7.03. Found: C, 72.43; $\mathrm{H}, 4.72 ; \mathrm{N}, 7.15$.

\section{Suzuki-Coupling Reactions of Pinacolboronate Esters 10 and 21; General Procedure B}

A $100 \mathrm{~mL}$ round-bottomed flask, equipped with a condenser and a magnetic stir bar, was charged with the corresponding bromoheteroaryl ( 1 equiv), $\mathrm{Na}_{2} \mathrm{CO}_{3}$ (2 equiv) and toluene-EtOH $(20 \mathrm{~mL}, 3: 1$ $\mathrm{v} / \mathrm{v}$ ). Thereafter, compound $\mathbf{1 0}$ or $\mathbf{2 1}$ (see below for the preparation of 21) (2.0 equiv) was added to the resulting suspension. The mixture obtained was degassed, purged with $\mathrm{N}_{2}$ for 15-20 min, and then $\mathrm{Pd}\left(\mathrm{PPh}_{3}\right)_{4}(10 \mathrm{~mol} \%)$ was added. The reaction mixture was stirred and heated at $90{ }^{\circ} \mathrm{C}$ for $8 \mathrm{~h}$. The mixture was allowed to cool to r.t., brine $(20 \mathrm{~mL})$ was added, and stirred for $30 \mathrm{~min}$. The organic layer was then diluted with EtOAc, transferred to a separatory funnel, washed with $\mathrm{H}_{2} \mathrm{O}$ and brine, dried $\left(\mathrm{Na}_{2} \mathrm{SO}_{4}\right)$, filtered, and concentrated under reduced pressure. The resulting residue was purified on silica gel to provide the title compound as an oil.

\section{2-[2-(Pyridin-2-yl)phenoxy]acetonitrile (13)}

The title compound was obtained by following General Procedure B from 2-bromopyridine $(0.157 \mathrm{~g}, 0.99 \mathrm{mmol})$ and $\mathbf{1 0}(0.309 \mathrm{~g}, 1.98$ $\mathrm{mmol})$ as a colorless oil; yield: $0.141 \mathrm{~g}(0.67 \mathrm{mmol}, 68 \%)$.

IR (neat): 3358, 3206, 1774, 1658, 1585, 1359, 1145, $854 \mathrm{~cm}^{-1}$. 
${ }^{1} \mathrm{H}$ NMR $\left(400 \mathrm{MHz}, \mathrm{CDCl}_{3}\right): \delta=4.75\left(\mathrm{~s}, 2 \mathrm{H}, \mathrm{CH}_{2}\right), 7.11$ (dd, $J=0.9,8.2$ Hz, 1 H, CH-6 Ar), 7.16-7.29 (m, 2 H, CH-4 Ar, CH-5 Pyr), 7.43 (ddd, $J=1.7,7.4,8.2 \mathrm{~Hz}, 1 \mathrm{H}, \mathrm{CH}-5 \mathrm{Ar}), 7.51-7.82(\mathrm{~m}, 3 \mathrm{H}, \mathrm{CH}-3 \mathrm{Ar}, \mathrm{CH}-3$ and CH-4 Pyr), 8.65-8.74 (m, 1 H, CH-6 Pyr).

${ }^{13} \mathrm{C} \mathrm{NMR}\left(100 \mathrm{MHz}, \mathrm{CDCl}_{3}\right): \delta=54.9\left(\mathrm{CH}_{2}\right), 114.5(\mathrm{C}-6 \mathrm{Ar}), 1148(\mathrm{CN})$, 121.5 (C-4 Pyr), 123.7 (C-4 Ar), 124.8 (C-6 Pyr), 125.3 (C-2 Ar), 128.6 (C-5 Ar), 129.7 (C-3 Ar), 135.3 (C-5 Pyr) 149.6 (C-3 Pyr), 152.6 (C-1 Ar) 157.6 (C-1 Pyr).

Anal. Calcd for $\mathrm{C}_{13} \mathrm{H}_{10} \mathrm{~N}_{2} \mathrm{O}: \mathrm{C}, 74.27 ; \mathrm{H}, 4.79 ; \mathrm{N}, 13.33$. Found: C, 74.31; $\mathrm{H}, 4.88$; N, 13.22.

\section{2-[2-(Pyridin-3-yl)phenoxy]acetonitrile (14)}

The title compound was obtained by following General Procedure B from 3-bromopyridine $(0.093 \mathrm{~mL}, 0.96 \mathrm{mmol})$ and $10(0.30 \mathrm{~g}, 1.16$ $\mathrm{mmol}$ ) as a colorless oil; yield: $0.096 \mathrm{~g}(0.45 \mathrm{mmol}, 40 \%)$.

IR (neat): 3375, 1663, 1637, 1559, 1394, 1349, 1127, 876, $808 \mathrm{~cm}^{-1}$.

${ }^{1} \mathrm{H}$ NMR $\left(400 \mathrm{MHz}, \mathrm{CDCl}_{3}\right): \delta=4.71\left(\mathrm{~s}, 2 \mathrm{H}, \mathrm{CH}_{2}\right), 7.12(\mathrm{dd}, J=0.9,8.2$ $\mathrm{Hz}, 1 \mathrm{H}, \mathrm{CH}-6 \mathrm{Ar}), 7.21$ (dt, J = 0.9, 8.6 Hz, $1 \mathrm{H}, \mathrm{CH}-4 \mathrm{Ar}), 7.30-7.52(\mathrm{~m}$, $3 \mathrm{H}, \mathrm{CH}-5 \mathrm{Pyr}, \mathrm{CH}-3$ and CH-5 Ar), 7.81 (dt, $J=1.8,7.9 \mathrm{~Hz}, 1 \mathrm{H}, \mathrm{CH}-4$ Ar), 8.59 (d, J = 1.8, 4.9 Hz, $1 \mathrm{H}, \mathrm{CH}-6 \mathrm{Pyr}), 8.72$ (dd, $J=0.8,2.3 \mathrm{~Hz}, 1 \mathrm{H}$, CH-2 Pyr).

${ }^{13} \mathrm{C}$ NMR $\left(100 \mathrm{MHz}, \mathrm{CDCl}_{3}\right): \delta=54.9\left(\mathrm{CH}_{2}\right), 114.5(\mathrm{C}-6 \mathrm{Ar}), 1148(\mathrm{CN})$, 122.8 (C-5 Pyr), 123.7 (C-4 Ar), 125.3 (C-2 Ar), 128.6 (C-5 Ar), 129.7 (C-3 Ar), 134.6 (C-1 Pyr), 136.9 (C-6 Pyr) 148.2 (C-4 Pyr), 150.2 (C-2 Pyr), 152.6 (C-1 Ar).

Anal. Calcd for $\mathrm{C}_{13} \mathrm{H}_{10} \mathrm{~N}_{2} \mathrm{O}: \mathrm{C}, 74.27 ; \mathrm{H}, 4.79 ; \mathrm{N}, 13.33$. Found: C, 74.43; $\mathrm{H}, 4.92 ; \mathrm{N}, 13.39$.

\section{$\mathbf{L i A l H}_{\mathbf{4}}$ Reduction of Nitriles; General Procedure C}

The selected nitrile derivative (1 equiv) was dissolved in of anhydrous $\mathrm{Et}_{2} \mathrm{O}\left(1-9 \mathrm{~mL}\right.$ ) and added dropwise to a suspension of $\mathrm{LiAlH}_{4}$ (3 equiv) in anhydrous $\mathrm{Et}_{2} \mathrm{O}(2 \mathrm{~mL})$ at $0{ }^{\circ} \mathrm{C}$. The excess of the reducing agent was immediately and carefully quenched with $\mathrm{H}_{2} \mathrm{O}$ and $5 \%$ aq $\mathrm{NaOH}$. The reaction mixture was stirred for $15 \mathrm{~min}$ and then EtOAc was added. The resulting organic phase was washed with brine, dried $\left(\mathrm{Na}_{2} \mathrm{SO}_{4}\right)$, filtered, and evaporated under reduced pressure. The crude product was used in the next synthetic step without any purification.

\section{2-[2-(Thiophen-2-yl)phenoxy]ethanamine (15)}

The title compound was obtained by following General Procedure $\mathrm{C}$ from $11(0.223 \mathrm{~g}, 1.03 \mathrm{mmol})$ as a colorless oil; yield: $0.208 \mathrm{~g}(0.94$ mmol, 92\%).

IR (neat): 3425 (br), 3248, 3085, 1859, 1641, 1388, 1365, 1101, 813, $708 \mathrm{~cm}^{-1}$.

${ }^{1} \mathrm{H} \mathrm{NMR}\left(400 \mathrm{MHz}, \mathrm{CDCl}_{3}\right): \delta=2.76\left(\mathrm{br} \mathrm{s}, 2 \mathrm{H}, \mathrm{NH}_{2}\right), 3.16(\mathrm{t}, J=5.0 \mathrm{~Hz}$, $\left.2 \mathrm{H}, \mathrm{CH}_{2} \mathrm{NH}_{2}\right), 4.08\left(\mathrm{t}, J=5.0 \mathrm{~Hz}, 2 \mathrm{H}, \mathrm{OCH}_{2}\right), 6.95(\mathrm{dd}, J=1.0,8.2 \mathrm{~Hz}, 1$ $\mathrm{H}, \mathrm{CH}-6 \mathrm{Ar}$ ), 7.02 (dt, J = 1.0, 7.6 Hz, $1 \mathrm{H}, \mathrm{CH}-4 \mathrm{Ar}$ ), 7.11 (dd, $J=3.7,5.1$ $\mathrm{Hz}, 1 \mathrm{H}, \mathrm{CH}-4$ Thio), 7.23-7.30 (m, $1 \mathrm{H}, \mathrm{CH}-5 \mathrm{Ar}$ ), 7.35 (dd, J = 1.1, 5.1 $\mathrm{Hz}, 1 \mathrm{H}, \mathrm{CH}-5$ Thio), 7.50 (dd, $J=1.1,3.7 \mathrm{~Hz}, 1 \mathrm{H}, \mathrm{CH}-3$ Thio), 7.69 (dd, $1 \mathrm{H}, J=1.7,7.6 \mathrm{~Hz}, \mathrm{CH}-3 \mathrm{Ar})$.

${ }^{13} \mathrm{C}$ NMR $\left(100 \mathrm{MHz}, \mathrm{CDCl}_{3}\right): \delta=47.4\left(\mathrm{CH}_{2} \mathrm{NH}_{2}\right), 70.6\left(\mathrm{OCH}_{2}\right), 112.5(\mathrm{C}-6$ Ar), 121.3 (C-4 Ar), 123.2 (C-2 Ar), 124.9 (C-3 Thio), 125.5 (C-5 Thio), 126.7 (C-4 Thio), 128.4 (C-5 Ar), 128.7 (C-3 Ar), 139.2 (C-1 Thio), $154.6(\mathrm{C}-1)$.

Anal. Calcd for $\mathrm{C}_{12} \mathrm{H}_{13}$ NOS: C, 65.72; H, 5.97; N, 6.39. Found: C, 65.83; H, 6.16; N, 6.67.

\section{2-[2-(Furan-2-yl)phenoxy]ethanamine (16)}

The title compound was obtained by following General Procedure $\mathrm{C}$ from $12(0.170 \mathrm{~g}, 0.85 \mathrm{mmol})$ as a yellow oil; yield: $0.103 \mathrm{~g}(0.50$ mmol, 60\%).

IR (neat): 3368 (br), 3251, 3146, 1859, 1667, 1354, 1093, 863, $706 \mathrm{~cm}^{-}$ 1 .

${ }^{1} \mathrm{H}$ NMR (400 MHz, $\left.\mathrm{CDCl}_{3}\right): \delta=1.40\left(\mathrm{br} \mathrm{s}, 2 \mathrm{H}, \mathrm{NH}_{2}\right), 3.20(\mathrm{t}, J=5.1 \mathrm{~Hz}$, $2 \mathrm{H}, \mathrm{CH}_{2} \mathrm{NH}_{2}$ ), 4.14 (t, $\left.J=5.1 \mathrm{~Hz}, 2 \mathrm{H}, \mathrm{OCH}_{2}\right), 6.50$ (dd, $J=1.7,3.2 \mathrm{~Hz}, 1$ $\mathrm{H}, \mathrm{CH}-4$ Fur), 6.94 (dd, $J=0.7,3.2 \mathrm{~Hz}, 1 \mathrm{H}, \mathrm{CH}-3 \mathrm{Fur}$ ), 6.96 (dd, $J=1.2$, $8.0 \mathrm{~Hz}, 1 \mathrm{H}, \mathrm{CH}-6 \mathrm{Ar}$ ), 7.04 (dt, J = 1.2, 7.6 Hz, $1 \mathrm{H}, \mathrm{CH}-4 \mathrm{Ar}$ ), 7.24 (ddd, $J=1.7,7.6,8.0 \mathrm{~Hz}, 1 \mathrm{H}, \mathrm{CH}-5 \mathrm{Ar}$ ), 7.48 (dd, $J=0.7,1.7 \mathrm{~Hz}, 1 \mathrm{H}, \mathrm{CH}-5$ Fur), 7.87 (dd, $J=1.7,7.6 \mathrm{~Hz}, 1 \mathrm{H}, \mathrm{CH}-3 \mathrm{Ar}$ ).

${ }^{13} \mathrm{C} \mathrm{NMR}\left(100 \mathrm{MHz}, \mathrm{CDCl}_{3}\right): \delta=47.6\left(\mathrm{CH}_{2} \mathrm{NH}_{2}\right), 70.7\left(\mathrm{OCH}_{2}\right), 109.9(\mathrm{C}-5$ Fur), 111.8 (C-4 Fur), 112.7 (C-6 Ar), 120.3 (C-2 Ar), 121.2 (C-4 Ar), 128.4 (C-5 Ar), 128.6 (C-3 Ar), 141.3 (C-3 Fur), 150.4 (C-1 Fur), 154.6 (C-1).

Anal. Calcd for $\mathrm{C}_{12} \mathrm{H}_{13} \mathrm{NO}_{2}$ : C, 70.92; $\mathrm{H}, 6.45 ; \mathrm{N}, 6.89$. Found: C, 70.96; $\mathrm{H}, 6.53 ; \mathrm{N}, 6.95$.

\section{2-[2-(Pyridin-2-yl)phenoxy]ethanamine (17)}

The title compound was obtained by following General Procedure C from $13(0.215 \mathrm{~g}, 1.02 \mathrm{mmol})$ as a yellow oil; yield: $0.195 \mathrm{~g}(0.91$ mmol, 79\%).

IR (neat): 3374 (br), 3236, 3171, 3101, 1853, 1781, 1625, 1390, 1153, $652 \mathrm{~cm}^{-1}$.

${ }^{1} \mathrm{H} \mathrm{NMR}\left(400 \mathrm{MHz}, \mathrm{CDCl}_{3}\right): \delta=1.60\left(\mathrm{br} \mathrm{s}, 2 \mathrm{H}, \mathrm{NH}_{2}\right), 3.03(\mathrm{t}, J=5.2 \mathrm{~Hz}$, $\left.2 \mathrm{H}, \mathrm{CH}_{2} \mathrm{NH}_{2}\right), 4.05\left(\mathrm{t}, J=5.2 \mathrm{~Hz}, 2 \mathrm{H}, \mathrm{OCH}_{2}\right), 7.02(\mathrm{dd}, J=1.0,8.4 \mathrm{~Hz}, 1$ $\mathrm{H}, \mathrm{CH}-6 \mathrm{Ar}$ ), 7.10 (dt, $J=1.0,7.6 \mathrm{~Hz}, 1 \mathrm{H}, \mathrm{CH}-4 \mathrm{Ar}), 7.16-7.25(\mathrm{~m}, 1 \mathrm{H}$, CH-5 Pyr), 7.31-7.40 (m, $1 \mathrm{H}, \mathrm{CH}-5 \mathrm{Ar}$ ), 7.62-7.83 (m, $3 \mathrm{H}, \mathrm{CH}-3 \mathrm{Ar}$, $\mathrm{CH}-3$ and $\mathrm{CH}-4 \mathrm{Pyr}$ ), 8.63 (d, $J=4.6 \mathrm{~Hz}, 1 \mathrm{H}, \mathrm{CH}-6 \mathrm{Pyr}$ ).

${ }^{13} \mathrm{C} \mathrm{NMR}\left(100 \mathrm{MHz}, \mathrm{CDCl}_{3}\right): \delta=52.2\left(\mathrm{CH}_{2} \mathrm{NH}_{2}\right), 68.9\left(\mathrm{OCH}_{2}\right), 113.1(\mathrm{C}-6$ Ar), 121.6 (C-4 Pyr), 121.9 (C-4 Ar), 124.9 (C-6 Pyr), 128.1 (C-2 Ar), 128.7 (C-5 Ar), 129.7 (C-3 Ar), 135.3 (C-5 Pyr) 149.3 (C-3 Pyr), 152.6 (C-1 Ar), 155.9 (C-1 Pyr).

Anal. Calcd for $\mathrm{C}_{13} \mathrm{H}_{14} \mathrm{~N}_{2} \mathrm{O}$ : C, 72.87; $\mathrm{H}, 6.59 ; \mathrm{N}, 13.07$. Found: C, 72.85; H, 6.69; N, 13.21 .

\section{2-[2-(Pyridin-3-yl)phenoxy]ethanamine (18)}

The title compound was obtained by following General Procedure $\mathrm{C}$ from $14(0.199 \mathrm{~g}, 0.95 \mathrm{mmol})$ as a yellow oil; yield: $0.180 \mathrm{~g}(0.84$ mmol, $79 \%)$.

IR (neat): 3372 (br), 3247, 3150, 1857, 1663, 1396, 1356, 1129, 711 $\mathrm{cm}^{-1}$.

${ }^{1} \mathrm{H} \mathrm{NMR}\left(400 \mathrm{MHz}, \mathrm{CDCl}_{3}\right): \delta=1.79\left(\mathrm{br} \mathrm{s}, 2 \mathrm{H}, \mathrm{NH}_{2}\right), 2.95(\mathrm{t}, J=5.2 \mathrm{~Hz}$, $\left.2 \mathrm{H}, \mathrm{CH}_{2} \mathrm{NH}_{2}\right), 3.93\left(\mathrm{t}, \mathrm{J}=5.2 \mathrm{~Hz}, 2 \mathrm{H}, \mathrm{OCH}_{2}\right), 6.93-7.06$ (m, $2 \mathrm{H}, \mathrm{CH}-4$, CH-6 Ar), 7.19-7.32 (m, 3 H, CH-5 Pyr, CH-3 and CH-5 Ar), 7.81 (dt, $J=1.8,7.9 \mathrm{~Hz}, 1 \mathrm{H}, \mathrm{CH}-4 \mathrm{Pyr}$ ), 8.51 (dd, $J=1.8,4.9$ Hz, $1 \mathrm{H}, \mathrm{CH}-6$ Pyr), 8.75 (dd, $J=0.9,2.4 \mathrm{~Hz}, 1 \mathrm{H}, \mathrm{CH}-2 \mathrm{Pyr}$ ).

${ }^{13} \mathrm{C} \mathrm{NMR}\left(100 \mathrm{MHz}, \mathrm{CDCl}_{3}\right): \delta=52.3\left(\mathrm{CH}_{2} \mathrm{NH}_{2}\right), 68.7\left(\mathrm{OCH}_{2}\right), 113.3(\mathrm{C}-6$ Ar), 121.9 (C-4 Ar), 122.6 (C-5 Pyr), 128.1 (C-2 Ar), 128.7 (C-5 Ar), 129.7 (C-3 Ar), 134.3 (C-1 Pyr), 136.8 (C-6 Pyr) 148.1 (C-4 Pyr), 150.1 (C-2 Pyr), 152.6 (C-1 Ar).

Anal. Calcd for $\mathrm{C}_{13} \mathrm{H}_{14} \mathrm{~N}_{2} \mathrm{O}$ : C, 72.87; $\mathrm{H}, 6.59 ; \mathrm{N}, 13.07$. Found: C, 72.96; H, 6.74; N, 13.36 . 


\section{tert-Butyl $\mathbf{N}$-(2-Chloroethyl)carbamate (19)}

In a round-bottomed flask, 2-chloroethylamine hydrochloride (2 g, $17.0 \mathrm{mmol})$ and $\mathrm{Et}_{3} \mathrm{~N}(2.37 \mathrm{~mL}, 11.0 \mathrm{mmol})$ were dissolved in anhydrous $\mathrm{CH}_{2} \mathrm{Cl}_{2}\left(24 \mathrm{~mL}\right.$ ). $\mathrm{Boc}_{2} \mathrm{O}$ ( $3.6 \mathrm{~mL}, 15.3 \mathrm{mmol}$ ) was added to the resulting solution under $\mathrm{N}_{2}$ at $0{ }^{\circ} \mathrm{C}$. The reaction mixture was stirred at r.t. for $4 \mathrm{~h}$. The mixture was then washed with $\mathrm{H}_{2} \mathrm{O}$ and brine, dried $\left(\mathrm{Na}_{2} \mathrm{SO}_{4}\right)$, filtered, and concentrated under reduced pressure to give the title product as a dark liquid; yield: $2.91 \mathrm{~g}$ (16.21 mmol, 99\%), which was used directly in the next synthetic step.

IR (neat): 3347, 2924, 2854, 2361, 2342, 1725, 1709, 1503, $1462 \mathrm{~cm}^{-1}$. ${ }^{1} \mathrm{H}$ NMR $\left(400 \mathrm{MHz}, \mathrm{CDCl}_{3}\right): \delta=? ?\left[\mathrm{~s}, 9 \mathrm{H}, \mathrm{C}\left(\mathrm{CH}_{3}\right)_{3}\right], 3.36-3.48(\mathrm{~m}, 2 \mathrm{H}$, $\mathrm{CH}_{2} \mathrm{NH}$ ), 3.49-3.63 (m, $\left.2 \mathrm{H}, \mathrm{ClCH}_{2}\right), 4.93$ (br s, $1 \mathrm{H}, \mathrm{NHCO}$ ).

${ }^{13} \mathrm{C}$ NMR $\left(100 \mathrm{MHz}, \mathrm{CDCl}_{3}\right): \delta=28.2\left[\mathrm{C}\left(\mathrm{CH}_{3}\right)_{3}\right], 32.8\left(\mathrm{CH}_{2} \mathrm{NH}\right), 45.7$ $\left(\mathrm{CH}_{2} \mathrm{Cl}\right), 79.8\left[\mathrm{C}\left(\mathrm{CH}_{3}\right)_{3}\right], 155.8(\mathrm{C}=\mathrm{O})$.

Anal. Calcd for $\mathrm{C}_{7} \mathrm{H}_{14} \mathrm{ClNO}_{2}$ : C, 46.80; H, 7.86; N, 7.80. Found: C, 46.91; $\mathrm{H}, 7.89 ; \mathrm{N}, 7.74$.

\section{tert-Butyl [2-(2-Iodophenoxy)ethyl]carbamate (20)}

Compound 19 ( $4.9 \mathrm{~g}, 27.27 \mathrm{mmol}$ ) and $\mathrm{KI}(7.55 \mathrm{~g}, 45.46 \mathrm{mmol})$ were added to a solution of $o$-iodophenol $(5.0 \mathrm{~g}, 22.73 \mathrm{mmol})$ in anhydrous DMF $(80 \mathrm{~mL})$ in the presence of $\mathrm{K}_{2} \mathrm{CO}_{3}(9.43 \mathrm{~g}, 68.2 \mathrm{mmol})$. The reaction mixture was magnetically stirred and refluxed for $4 \mathrm{~h}$. Then the reaction mixture was cooled to r.t. and the solvent was evaporated under reduced pressure. The resulting residue was dissolved in EtOAc, and the EtOAc layer was washed with $\mathrm{H}_{2} \mathrm{O}$ and brine, dried $\left(\mathrm{Na}_{2} \mathrm{SO}_{4}\right)$, filtered, and concentrated under reduced pressure to afford the title compound as a dark oil; yield: $8.34 \mathrm{~g}$ (22.97 mmol, 98\%).

IR (neat): 3268, 1858, 1672, 1641, 1385, 1347, 1138, $1075 \mathrm{~cm}^{-1}$.

${ }^{1} \mathrm{H}$ NMR $\left(400 \mathrm{MHz}, \mathrm{CDCl}_{3}\right): \delta=1.45\left[\mathrm{~s}, 9 \mathrm{H}, \mathrm{C}\left(\mathrm{CH}_{3}\right)_{3}\right], 3.59(\mathrm{q}, 2 \mathrm{H}$, $\left.J=5.3 \mathrm{~Hz}, \mathrm{CH}_{2} \mathrm{NH}\right), 4.06\left(\mathrm{t}, J=4.9 \mathrm{~Hz}, 2 \mathrm{H}, \mathrm{OCH}_{2}\right), 6.13(\mathrm{br} \mathrm{s}, 1 \mathrm{H}, \mathrm{NH})$, $6.73(\mathrm{dt}, J=1.5,7.6 \mathrm{~Hz}, 1 \mathrm{H}, \mathrm{CH}-4 \mathrm{Ar}), 6.81(\mathrm{dd}, J=1.5,8.2 \mathrm{~Hz}, 1 \mathrm{H}, \mathrm{CH}-$ $6 \mathrm{Ar}$ ), 7.29 (ddd, $J=1.5,7.6,8.2 \mathrm{~Hz}, 1 \mathrm{H}, \mathrm{CH}-5 \mathrm{Ar}$ ), 7.77 (dd, $J=1.5,7.8$ $\mathrm{Hz}, 1 \mathrm{H}, \mathrm{CH}-3 \mathrm{Ar})$.

${ }^{13} \mathrm{C}$ NMR $\left(100 \mathrm{MHz}, \mathrm{CDCl}_{3}\right): \delta=28.2\left[\mathrm{C}\left(\mathrm{CH}_{3}\right)_{3}\right], 39.8\left(\mathrm{CH}_{2} \mathrm{NH}\right), 68.4$ $\left(\mathrm{OCH}_{2}\right), 79.2\left[\mathrm{C}\left(\mathrm{CH}_{3}\right)_{3}\right], 86.6(\mathrm{C}-2 \mathrm{Ar}), 112.3(\mathrm{C}-6 \mathrm{Ar}), 122.8(\mathrm{C}-4 \mathrm{Ar})$, 129.4 (C-5 Ar), 139.1 (C-3 Ar), 155.7 (C-1 Ar), $156.8\left(\mathrm{NHCO}_{2}\right)$.

Anal. Calcd for $\mathrm{C}_{13} \mathrm{H}_{18} \mathrm{INO}_{3}$ : C, 42.99; $\mathrm{H}, 5.00 ; \mathrm{N}, 3.86$. Found: $\mathrm{C}, 43.23$; H, 5.18; N, 3.96.

\section{tert-Butyl \{2-[2-(4,4,5,5-Tetramethyl-1,3,2-dioxaborlan-2-yl)phe-} noxy]ethyl\}carbamate (21)

Pinacolborane $(0.387 \mathrm{~mL}, 2.66 \mathrm{mmol})$ and $\mathrm{Et}_{3} \mathrm{~N}(0.574 \mathrm{~mL}, 4.12$ $\mathrm{mmol})$ were added to a solution of $\mathbf{2 0}(0.372 \mathrm{~g}, 1.03 \mathrm{mmol})$ in anhydrous 1,4-dioxane $(10 \mathrm{~mL})$ under $\mathrm{N}_{2}$. The mixture was degassed, purged with $\mathrm{N}_{2}$ for $10 \mathrm{~min}$, and $\mathrm{Pd}(\mathrm{OAc})_{2}(0.015 \mathrm{~g}, 0.05 \mathrm{mmol}, 5 \mathrm{~mol} \%)$ and (2-biphenyl)dicyclohexylphosphine (0.098 g, $0.2 \mathrm{mmol}, 20 \mathrm{~mol} \%$ ) were added. The mixture was vigorously stirred at $100{ }^{\circ} \mathrm{C}$ under $\mathrm{N}_{2}$ for $2 \mathrm{~h}$. The reaction mixture was allowed to cool to r.t., carefully quenched with sat. aq $\mathrm{NH}_{4} \mathrm{Cl}$ at $0{ }^{\circ} \mathrm{C}$, and extracted with $\mathrm{CH}_{2} \mathrm{Cl}_{2}$. The combined organic layers were dried $\left(\mathrm{Na}_{2} \mathrm{SO}_{4}\right)$, filtered, and evaporated under reduced pressure. The crude product was purified by flash column chromatography to give the title compound as a yellow oil; yield: $0.216 \mathrm{~g}(0.59 \mathrm{mmol}, 99 \%)$.

IR (neat): 3295 (br), 3200, 1836, 1710, 1503, 1345, $841 \mathrm{~cm}^{-1}$.

${ }^{1} \mathrm{H} \mathrm{NMR}\left(400 \mathrm{MHz}, \mathrm{CDCl}_{3}\right): \delta=1.39\left[\mathrm{~s}, 9 \mathrm{H}, \mathrm{C}\left(\mathrm{CH}_{3}\right)_{3}\right], 1.47[\mathrm{~s}, 12 \mathrm{H}, 2 \times$ $\left.\mathrm{C}\left(\mathrm{CH}_{3}\right)_{2}\right], 3.59\left(\mathrm{q}, \mathrm{J}=5.1 \mathrm{~Hz}, 2 \mathrm{H}, \mathrm{CH}_{2} \mathrm{NH}\right), 4.06\left(\mathrm{t}, J=5.1 \mathrm{~Hz}, 2 \mathrm{H}, \mathrm{OCH}_{2}\right)$, 6.18 (br s, $1 \mathrm{H}, \mathrm{NH}$ ), 6.85 (d, $J=8.3 \mathrm{~Hz}, 1 \mathrm{H}, \mathrm{CH}-6 \mathrm{Ar}$ ), 6.98 (dt, $J=0.9$, $7.4 \mathrm{~Hz}, 1 \mathrm{H}, \mathrm{CH}-4 \mathrm{Ar}$ ), 7.43 (ddd, $J=1.8,7.4,8.3 \mathrm{~Hz}, 1 \mathrm{H}, \mathrm{CH}-5 \mathrm{Ar}$ ), 7.71 (dd, $J=1.8,7.4 \mathrm{~Hz}, 1 \mathrm{H}, \mathrm{CH}-3 \mathrm{Ar}$ ).
${ }^{13} \mathrm{C} \mathrm{NMR}\left(100 \mathrm{MHz}, \mathrm{CDCl}_{3}\right): \delta=24.9\left[2 \times \mathrm{C}\left(\mathrm{CH}_{3}\right)_{2}\right], 28.4\left[\mathrm{C}\left(\mathrm{CH}_{3}\right)_{3}\right], 39.9$ $\left(\mathrm{CH}_{2} \mathrm{NH}\right), 68.3\left(\mathrm{OCH}_{2}\right), 79.4\left[\mathrm{C}\left(\mathrm{CH}_{3}\right)_{3}\right], 83.6\left[2 \times \mathrm{C}\left(\mathrm{CH}_{3}\right)_{2}\right], 114.9(\mathrm{C}-6$ $\mathrm{Ar}), 115.7$ (C-2 Ar), 123.2 (C-4 Ar), 131.8 (C-5 Ar), 138.2 (C-3 Ar), $156.7\left(\mathrm{NHCO}_{2}\right), 160.9(\mathrm{C}-1 \mathrm{Ar})$.

Anal. Calcd for $\mathrm{C}_{19} \mathrm{H}_{30} \mathrm{BNO}_{5}$ : C, 62.82; $\mathrm{H}, 8.32 ; \mathrm{N}, 3.86$. Found: $\mathrm{C}, 63.02$; $\mathrm{H}, 8.41 ; \mathrm{N}, 3.71$.

tert-Butyl N-\{2-[2-(Pyrimidin-2-yl)phenoxy]ethyl\}carbamate (22) The title compound was obtained by following General Procedure B from 2-bromopyrimidine (0.238 g, $1.5 \mathrm{mmol})$ and 21 (0.694 g, 1.90 $\mathrm{mmol})$ as a dark oil; yield: $0.171 \mathrm{~g}, 0.54 \mathrm{mmol}(34 \%)$.

IR (neat): 3279, 1854, 1740, 1520, 1441, 1375, $1024 \mathrm{~cm}^{-1}$.

${ }^{1} \mathrm{H} \mathrm{NMR}\left(400 \mathrm{MHz}, \mathrm{CDCl}_{3}\right): \delta=1.36\left[\mathrm{~s}, 9 \mathrm{H}, \mathrm{C}\left(\mathrm{CH}_{3}\right)_{3}\right], 3.51(\mathrm{t}, J=4.9 \mathrm{~Hz}$, $\left.2 \mathrm{H}, \mathrm{CH}_{2} \mathrm{NH}\right), 4.25\left(\mathrm{t}, J=4.9 \mathrm{~Hz}, 2 \mathrm{H}, \mathrm{CH}_{2} \mathrm{O}\right), 5.29(\mathrm{br} \mathrm{s}, 1 \mathrm{H}, \mathrm{NH}), 7.06$ (d, $J=8.3 \mathrm{~Hz}, 1 \mathrm{H}, \mathrm{CH}-6 \mathrm{Ar}), 7.08-7.13$ (m, $1 \mathrm{H}, \mathrm{CH}-4 \mathrm{Ar}), 7.25$ (t, $J=4.9$ $\mathrm{Hz}, 1 \mathrm{H}, \mathrm{CH}-5$ Pyrim), 7.39-7.48 (m, $1 \mathrm{H}, \mathrm{CH}-5 \mathrm{Ar}), 7.82-7.91(\mathrm{~m}, 1 \mathrm{H}$, CH-3 Ar), 8.92 (d, J = 4.9 Hz, $2 \mathrm{H}, \mathrm{CH}-4, \mathrm{CH}-6$ Pyrim).

${ }^{13} \mathrm{C}$ NMR $\left(100 \mathrm{MHz}, \mathrm{CDCl}_{3}\right): \delta=28.3\left[\mathrm{C}\left(\mathrm{CH}_{3}\right)_{3}\right], 40.2\left(\mathrm{CH}_{2} \mathrm{NH}\right), 68.1$

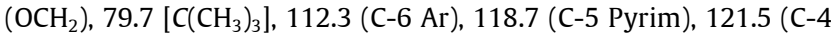
$\mathrm{Ar}), 128.1$ (C-2 Ar), 129.6 (C-5 Ar), 131.3 (C-3 Ar), 154.4 (C-1 Ar), 156.9 (C-4, C-6 Pyrim), 155.9 (C=0), 165.96 (C-2 Pyrim).

Anal. Calcd for $\mathrm{C}_{17} \mathrm{H}_{21} \mathrm{~N}_{3} \mathrm{O}_{3}$ : C, 64.74; $\mathrm{H}, 6.71 ; \mathrm{N}, 13.32$. Found: $\mathrm{C}$, 64.62; $\mathrm{H}, 6.41 ; \mathrm{N}, 13.61$.

tert-Butyl N-\{2-[2-(Pyrimidin-5-yl)phenoxy]ethyl\}carbamate (23) The title compound was obtained by following General Procedure B from 5-bromopyrimidine $(0.238 \mathrm{~g}, 1.5 \mathrm{mmol})$ and $21(0.694 \mathrm{~g}, 1.90$ $\mathrm{mmol})$ as a dark oil; yield: $0.206 \mathrm{~g}(0.65 \mathrm{mmol}, 42 \%)$.

IR (neat): 3277 (br), 1847, 1670, 1552, 1343, 883, $686 \mathrm{~cm}^{-1}$.

${ }^{1} \mathrm{H} \mathrm{NMR}\left(400 \mathrm{MHz}, \mathrm{CDCl}_{3}\right): \delta=1.42\left[\mathrm{~s}, 9 \mathrm{H}, \mathrm{C}\left(\mathrm{CH}_{3}\right)_{3}\right], 3.46(\mathrm{t}, J=5.4 \mathrm{~Hz}$, $\left.2 \mathrm{H}, \mathrm{CH}_{2} \mathrm{NH}\right), 4.09\left(\mathrm{t}, J=5.2 \mathrm{~Hz}, 2 \mathrm{H}, \mathrm{OCH}_{2}\right), 4.72(\mathrm{br} \mathrm{s}, 1 \mathrm{H}, \mathrm{NH}), 7.04$ (dd, $J=1.2,8.3 \mathrm{~Hz}, \mathrm{CH}-6 \mathrm{Ar}$ ), $7.12(\mathrm{td}, J=1.2,7.4 \mathrm{~Hz}, 1 \mathrm{H}, \mathrm{CH}-4 \mathrm{Ar}$ ), 7.35 (dd, $J=1.8,7.4 \mathrm{~Hz}, 1 \mathrm{H}, \mathrm{CH}-3 \mathrm{Ar}$ ), 7.42 (ddd, $J=1.8,7.4,8.3 \mathrm{~Hz}, 1$ H, CH-5 Ar), 8.89 (s, 2 H, CH-4, CH-6 Pyrim), 9.17 (s, 1 H, CH-2 Pyrim). ${ }^{13} \mathrm{C}$ NMR $\left(100 \mathrm{MHz}, \mathrm{CDCl}_{3}\right): \delta=28.3\left[\mathrm{C}\left(\mathrm{CH}_{3}\right)_{3}\right], 40.2\left(\mathrm{CH}_{2} \mathrm{NH}\right), 68.3$ $\left(\mathrm{OCH}_{2}\right), 79.9\left[\mathrm{C}\left(\mathrm{CH}_{3}\right)_{3}\right], 112.3$ (C-6 Ar), $121.5(\mathrm{C}-4 \mathrm{Ar}), 124.4(\mathrm{C}-3 \mathrm{Ar})$, 130.2 (C-2 Ar), 130.6 (C-5 Ar), 132.2 (C-5 Pyrim), 154.4 (C-1 Ar), 155.9 (C=0), 156.5 (C-4, C-6 Pyrim), 156.8 (C-2 Pyrim).

Anal. Calcd for $\mathrm{C}_{17} \mathrm{H}_{21} \mathrm{~N}_{3} \mathrm{O}_{3}$ : C, 64.74; $\mathrm{H}, 6.71 ; \mathrm{N}, 13.32$. Found: $\mathrm{C}$, 64.60; H, 6.49; N, 13.56 .

\section{tert-Butyl $N$-\{2-[2-(1,3-Thiazol-2-yl)phenoxy]ethyl\}carbamate} (24)

The title compound was obtained by following General Procedure B from 2-bromothiazole $(0.060 \mathrm{~g}, 0.34 \mathrm{mmol})$ and $21(0.174 \mathrm{~g}, 0.48$ $\mathrm{mmol})$ as a dark oil; yield: $0.095 \mathrm{~g}(0.30 \mathrm{mmol}, 88 \%)$.

IR (neat): 3291, 3200, 1830, 1716, 1438, 1352, 1052, $876 \mathrm{~cm}^{-1}$.

$\left.{ }^{1} \mathrm{H} \mathrm{NMR} \mathrm{(400} \mathrm{MHz,} \mathrm{CDCl}_{3}\right): \delta=1.43\left[\mathrm{~s}, 9 \mathrm{H}, \mathrm{C}\left(\mathrm{CH}_{3}\right)_{3}\right], 3.73(\mathrm{t}, J=5.2 \mathrm{~Hz}$, $2 \mathrm{H}, \mathrm{CH}_{2} \mathrm{NH}$ ), 4.27 (t, J = 5.2 Hz, $2 \mathrm{H}, \mathrm{OCH}_{2}$ ), 5.25 (br s, $\left.1 \mathrm{H}, \mathrm{NH}\right), 7.02$ (dd, $J=1.0,8.3 \mathrm{~Hz}, 1 \mathrm{H}, \mathrm{CH}-6 \mathrm{Ar}$ ), $7.12(\mathrm{dt}, J=1.0,7.9 \mathrm{~Hz}, 1 \mathrm{H}, \mathrm{CH}-4$ Ar), 7.40 (ddd, $J=1.8,7.9,8.3 \mathrm{~Hz}, 1 \mathrm{H}, \mathrm{CH}-5 \mathrm{Ar}$ ), 7.42 (d, $J=3.4 \mathrm{~Hz}, 1$ H, CH-5 Thia), 7.94 (d, $J=3.4 \mathrm{~Hz}, 1 \mathrm{H}, \mathrm{CH}-4$ Thia), 8.40 (dd, $J=1.8,7.9$ $\mathrm{Hz}, 1 \mathrm{H}, \mathrm{CH}-3 \mathrm{Ar})$.

${ }^{13} \mathrm{C}$ NMR $\left(100 \mathrm{MHz}, \mathrm{CDCl}_{3}\right): \delta=28.6\left[\mathrm{C}\left(\mathrm{CH}_{3}\right)_{3}\right], 40.3\left(\mathrm{CH}_{2} \mathrm{NH}\right), 68.2$ $\left(\mathrm{OCH}_{2}\right), 79.7\left[\mathrm{C}\left(\mathrm{CH}_{3}\right)_{3}\right], 112.1$ (C-6 Ar), 119.5 (C-5 Thia), $121.4(\mathrm{C}-4 \mathrm{Ar})$, 122.1 (C-2 Ar), 128.7 (C-3 Ar), 130.9 (C-5 Ar), 142.1 (C-4 Thia), 155.5 (C-1 Ar), 155.9 (C=0), 162.1 (C-2 Thia). 
Anal. Calcd for $\mathrm{C}_{16} \mathrm{H}_{20} \mathrm{~N}_{2} \mathrm{O}_{3} \mathrm{~S}$ : C, 59.98; H, 6.29; N, 8.74. Found: $\mathrm{C}$, $60.12 ; \mathrm{H}, 6.37$; N, 8.91.

\section{tert-Butyl $N$-\{2-[2-(1,3-Thiazol-5-yl)phenoxy]ethyl\}carbamate (25)}

The title compound was obtained by following the General Procedure B from 5-bromothiazole $(0.087 \mathrm{~g}, 0.64 \mathrm{mmol})$ and $21(0.192 \mathrm{~g}, 0.53$ mmol) as a dark oil; yield: $0.057 \mathrm{~g}(0.18 \mathrm{mmol}, 33 \%)$.

IR (neat): 3291, 1832, 1708, 1467, 1395, 1338, $880 \mathrm{~cm}^{-1}$.

${ }^{1} \mathrm{H}$ NMR $\left(400 \mathrm{MHz}, \mathrm{CDCl}_{3}\right): \delta=1.49\left[\mathrm{~s}, 9 \mathrm{H}, \mathrm{C}\left(\mathrm{CH}_{3}\right)_{3}\right], 3.60-3.74(\mathrm{~m}, 2$ $\mathrm{H}, \mathrm{CH}_{2} \mathrm{NH}$ ), $4.20\left(\mathrm{t}, J=5.0 \mathrm{~Hz}, 2 \mathrm{H}, \mathrm{OCH}_{2}\right), 5.04$ (br s, $\left.1 \mathrm{H}, \mathrm{NH}\right), 7.02$ (d, $J=8.3 \mathrm{~Hz}, 1 \mathrm{H}, \mathrm{CH}-6 \mathrm{Ar}), 7.09$ (dt, $J=1.0,7.6 \mathrm{~Hz}, 1 \mathrm{H}, \mathrm{CH}-4 \mathrm{Ar}), 7.36$ (ddd, $J=1.5,7.6,8.3 \mathrm{~Hz}, 1 \mathrm{H}, \mathrm{CH}-5 \mathrm{Ar}$ ), 7.71 (dd, $J=1.5,7.6 \mathrm{~Hz}, 1 \mathrm{H}$, CH-3 Ar), 8.29 (s, $1 \mathrm{H}, \mathrm{CH}-4$ Thia), 8.85 (s, $1 \mathrm{H}, \mathrm{CH}-2$ Thia).

${ }^{13} \mathrm{C}$ NMR $\left(100 \mathrm{MHz}, \mathrm{CDCl}_{3}\right): \delta=28.4\left[\mathrm{C}\left(\mathrm{CH}_{3}\right)_{3}\right], 40.1\left(\mathrm{CH}_{2} \mathrm{NH}\right), 68.1$

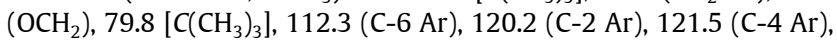
128.8 (C-3 Ar), 129.6 (C-5 Ar), 133.8 (C-5 Thia), 140.9 (C-4 Thia), 152.9 (C-2 Thia), 154.4 (C-1 Ar), 155.9 ( $\mathrm{C}=0$ ).

Anal. Calcd for $\mathrm{C}_{16} \mathrm{H}_{20} \mathrm{~N}_{2} \mathrm{O}_{3} \mathrm{~S}$ : C, 59.98; H, 6.29; N, 8.74. Found: C, 59.82; H, 6.39; N, 8.89.

\section{tert-Butyl $N$-\{2-[2-(1-Methyl-1H-pyrazol-4-yl)phenoxy]ethyl\}car- bamate (26)}

The title compound was obtained by following the General Procedure B from 4-bromo-1-methyl-1H-pyrazole $(0.084 \mathrm{~g}, 0.52 \mathrm{mmol})$ and 21 (0.190 g, $0.52 \mathrm{mmol})$ as a dark oil; yield: $0.090 \mathrm{~g}(0.28 \mathrm{mmol}, 54 \%)$.

IR (neat): 3291 (br), 1809, 1708, 1442, 1385, $893 \mathrm{~cm}^{-1}$.

${ }^{1} \mathrm{H}$ NMR $\left(400 \mathrm{MHz}, \mathrm{CDCl}_{3}\right): \delta=1.49\left[\mathrm{~s}, 9 \mathrm{H}, \mathrm{C}\left(\mathrm{CH}_{3}\right)_{3}\right], 3.60-3.75(\mathrm{~m}, 2$ $\left.\mathrm{H}, \mathrm{CH}_{2} \mathrm{NH}\right), 4.00\left(\mathrm{~s}, 3 \mathrm{H}, \mathrm{NCH}_{3}\right), 4.16\left(\mathrm{t}, J=5.2 \mathrm{~Hz}, 2 \mathrm{H}, \mathrm{OCH}_{2}\right), 4.90(\mathrm{br}$ s, $1 \mathrm{H}, \mathrm{NH}$ ), 6.96 (d, J = 7.6 Hz, $1 \mathrm{H}, \mathrm{CH}-6 \mathrm{Ar}), 7.04$ (dt, $J=0.9,7.5 \mathrm{~Hz}, 1$ H, CH-4 Ar), 7.17-7.26 (m, 1 H, CH-5 Ar), 7.56 (dd, J = 1.6, 7.6 Hz, 1 H, CH-3 Ar), 7.89 (s, 1 H, CH-5 Pyra), 7.90 (s, 1 H, CH-3 Pyra).

${ }^{13} \mathrm{C}$ NMR $\left(100 \mathrm{MHz}, \mathrm{CDCl}_{3}\right): \delta=28.4\left[\mathrm{C}\left(\mathrm{CH}_{3}\right)_{3}\right], 39.0\left(\mathrm{NCH}_{3}\right), 40.3$ $\left(\mathrm{CH}_{2} \mathrm{NH}\right), 67.6\left(\mathrm{OCH}_{2}\right), 79.6\left[\mathrm{C}\left(\mathrm{CH}_{3}\right)_{3}\right], 112.4(\mathrm{C}-6 \mathrm{Ar}), 118.5(\mathrm{C}-4$ Pyra $)$, 121.4 (C-4 Ar), 121.8 (C-2 Ar), 127.3 (C-5 Ar), 127.9 (C-3 Ar), 129.4 (C5 Pyra), 138.1 (C-3 Pyra), 154.7 (C-1 Ar), 155.9 (C=O).

Anal. Calcd for $\mathrm{C}_{17} \mathrm{H}_{23} \mathrm{~N}_{3} \mathrm{O}_{3}$ : C, 64.33; H, 7.30; N, 13.24. Found: $\mathrm{C}$, 64.26; H, 7.21; N, 13.39 .

\section{tert-Butyl $N$-\{2-[2-(1-Methyl-1H-imidazol-5-yl)phenoxy]eth- yl\}carbamate (27)}

The title compound was obtained by following the General Procedure B from 5-bromo-1-methyl-1H-imidazole ( $0.391 \mathrm{~g}, 2.44 \mathrm{mmol})$ and 21 (0.444 g, $1.22 \mathrm{mmol})$ as an oil; yield: $0.294 \mathrm{~g}(0.93 \mathrm{mmol}, 76 \%)$.

IR (neat): 3215 (br), 1833, 1707, 1445, 1338, $1123 \mathrm{~cm}^{-1}$.

${ }^{1} \mathrm{H}$ NMR $\left(400 \mathrm{MHz}, \mathrm{CDCl}_{3}\right): \delta=1.42\left[\mathrm{~s}, 9 \mathrm{H}, \mathrm{C}\left(\mathrm{CH}_{3}\right)_{3}\right], 3.40(\mathrm{~m}, 2 \mathrm{H}$, $\mathrm{CH}_{2} \mathrm{NH}$ ), $3.51\left(\mathrm{~s}, 3 \mathrm{H}, \mathrm{NCH}_{3}\right), 4.03\left(\mathrm{t}, J=5.2 \mathrm{~Hz}, 2 \mathrm{H}, \mathrm{OCH}_{2}\right), 4.84$ (br s, 1 $\mathrm{H}, \mathrm{NH}), 6.90-7.06$ ( $\mathrm{m}, 3 \mathrm{H}, \mathrm{CH}-4$ and CH-6 Ar, CH-4 Imi), 7.21-7.31 (m, $1 \mathrm{H}, \mathrm{CH}-5 \mathrm{Ar}$ ), 7.33-7.42 (m, $1 \mathrm{H}, \mathrm{CH}-3 \mathrm{Ar}$ ), 7.50 (s, $1 \mathrm{H}, \mathrm{CH}-2 \mathrm{Imi})$.

${ }^{13} \mathrm{C} \mathrm{NMR}\left(100 \mathrm{MHz}, \mathrm{CDCl}_{3}\right): \delta=28.5\left[\mathrm{C}\left(\mathrm{CH}_{3}\right)_{3}\right], 32.4\left(\mathrm{NCH}_{3}\right), 40.4$ $\left(\mathrm{CH}_{2} \mathrm{NH}\right), 67.8\left(\mathrm{OCH}_{2}\right), 79.7\left[\mathrm{C}\left(\mathrm{CH}_{3}\right)_{3}\right], 112.3(\mathrm{C}-6 \mathrm{Ar}), 121.5(\mathrm{C}-4 \mathrm{Ar})$, 121.7 (C-2 Ar), 127.3 (C-5 Ar), 127.9 (C-3 Ar), 128.6 (C-4 Imi), 129.9 (C-5 Imi), 138.5 (C-2 Imi), 154.7 (C-1 Ar), 155.9 (C=O).

Anal. Calcd for $\mathrm{C}_{17} \mathrm{H}_{23} \mathrm{~N}_{3} \mathrm{O}_{3}$ : C, 64.33; H, 7.30; N, 13.24. Found: C, $64.45 ; \mathrm{H}, 7.51 ; \mathrm{N}, 13.46$.

\section{Hydrolysis of Boc Esters; General Procedure D}

A $25 \mathrm{~mL}$ round-bottomed flask was charged with the selected NHBoc derivative (1 equiv) and $\mathrm{EtOH}(5 \mathrm{~mL})$. The solution was cooled to $0{ }^{\circ} \mathrm{C}$ in an ice water bath and $1.25 \mathrm{M} \mathrm{HCl}$ in $\mathrm{EtOH}$ (15 equiv) solution was added dropwise. The reaction was allowed to warm to r.t. and refluxed for $2 \mathrm{~h}$. The reaction was monitored by TLC (eluent: ????). The resulting suspension was cooled to r.t. and the solvent was removed under reduced pressure. The crude solid product was dissolved in $\mathrm{H}_{2} \mathrm{O}$ and then aq $5 \% \mathrm{NaOH}$ was added until $\mathrm{pH} 12$. The resulting solution was extracted with $\mathrm{CH}_{2} \mathrm{Cl}_{2}$. The combined organic layers were dried $\left(\mathrm{Na}_{2} \mathrm{SO}_{4}\right)$, filtered, and concentrated under reduced pressure to afford the corresponding pure product.

\section{2-[2-(Pyrimidin-2-yl)phenoxy]ethanamine (28)}

The title compound was obtained by following General Procedure D from $22(0.171 \mathrm{~g}, 0.54 \mathrm{mmol})$ as a dark oil; yield: $0.092 \mathrm{~g}$ (0.42 mmol, $82 \%)$.

IR (neat): 3380, 1733, 1709, 1596, 1401, 1397, 985, 907, $715 \mathrm{~cm}^{-1}$.

${ }^{1} \mathrm{H} \mathrm{NMR}\left(400 \mathrm{MHz}, \mathrm{CDCl}_{3}\right.$ ): $\delta=1.79$ (br s, $2 \mathrm{H}$ ), $3.00(\mathrm{t}, J=4.8 \mathrm{~Hz}, 2 \mathrm{H}$, $\left.\mathrm{CH}_{2} \mathrm{NH}_{2}\right), 4.07\left(\mathrm{t}, J=4.8 \mathrm{~Hz}, 2 \mathrm{H}, \mathrm{OCH}_{2}\right), 7.04(\mathrm{~d}, J=8.3 \mathrm{~Hz}, 1 \mathrm{H}, \mathrm{CH}-6$ $\mathrm{Ar}), 7.09$ (td, $J=0.9,7.5 \mathrm{~Hz}, 1 \mathrm{H}, \mathrm{CH}-4 \mathrm{Ar}), 7.22(\mathrm{t}, J=4.9 \mathrm{~Hz}, 1 \mathrm{H}, \mathrm{CH}-5$ Pyrim), 7.42 (ddd, $J=1.8,7.5,8.3 \mathrm{~Hz}, 1 \mathrm{H}, \mathrm{CH}-5 \mathrm{Ar}$ ), 7.79 (dd, $J=1.8$ $7.5 \mathrm{~Hz}, 1 \mathrm{H}, \mathrm{CH}-3 \mathrm{Ar}$ ), 8.85 (d, J = 4.9 Hz, $2 \mathrm{H}, \mathrm{CH}-4$, CH-6 Pyrim).

${ }^{13} \mathrm{C}$ NMR $\left(100 \mathrm{MHz}, \mathrm{CDCl}_{3}\right): \delta=41.6\left(\mathrm{CH}_{2} \mathrm{NH}_{2}\right), 71.3\left(\mathrm{OCH}_{2}\right), 113.7(\mathrm{C}-6$ $\mathrm{Ar}$ ), 118.7 (C-5 Pyrim), 121.1 (C-4 Ar), 128.6 (C-2 Ar), 131.1 (C-5 Ar), 131.7 (C-3 Ar), 156.9 (C-4, C-6 Pyrim), 157.1 (C-1 Ar), 165.96 (C-2 Pyrim).

Anal. Calcd for $\mathrm{C}_{12} \mathrm{H}_{13} \mathrm{~N}_{3} \mathrm{O}$ : C, 66.96; $\mathrm{H}, 6.09 ; \mathrm{N}, 19.52$. Found: C, 66.90; H, 6.00; N, 19.66.

\section{2-[2-(Pyrimidin-5-yl)phenoxy]ethanamine (29)}

The title compound was obtained by following General Procedure D from $23(0.181 \mathrm{~g}, 0.57 \mathrm{mmol})$ as a dark oil; yield: $0.089 \mathrm{~g}(0.41 \mathrm{mmol}$, $72 \%)$.

IR (neat): 3248, 3166, 1677, 1563, 1405, 1387, 899, $818 \mathrm{~cm}^{-1}$.

${ }^{1} \mathrm{H} \mathrm{NMR} \mathrm{(400} \mathrm{MHz,} \mathrm{CDCl} 3$ ): $\delta=1.50\left(\right.$ br s, $\left.2 \mathrm{H} \mathrm{NH}_{2}\right), 3.05(\mathrm{t}, J=5.3 \mathrm{~Hz}$, $\left.2 \mathrm{H}, \mathrm{CH}_{2} \mathrm{NH}_{2}\right), 4.04\left(\mathrm{t}, J=5.3 \mathrm{~Hz}, 2 \mathrm{H}, \mathrm{OCH}_{2}\right), 7.05(\mathrm{dd}, J=0.9,8.3 \mathrm{~Hz}, 1$ $\mathrm{H}, \mathrm{CH}-6 \mathrm{Ar}$ ), 7.11 (td, $J=0.9,7.5 \mathrm{~Hz}, 1 \mathrm{H}, \mathrm{CH}-4 \mathrm{Ar}$ ), 7.35 (dd, $J=1.8,7.5$ $\mathrm{Hz}, 1 \mathrm{H}, \mathrm{CH}-3 \mathrm{Ar}$ ), 7.42 (ddd, J = 1.8, 7.5, $8.3 \mathrm{~Hz}, 1 \mathrm{H}, \mathrm{CH}-5 \mathrm{Ar}$ ), 8.93 (s, $2 \mathrm{H}, \mathrm{CH}-4, \mathrm{CH}-6$ Pyrim), 9.17 (s, $1 \mathrm{H}, \mathrm{CH}-2$ Pyrim).

${ }^{13} \mathrm{C} \mathrm{NMR}\left(100 \mathrm{MHz}, \mathrm{CDCl}_{3}\right): \delta=41.4\left(\mathrm{CH}_{2} \mathrm{NH}_{2}\right), 70.8\left(\mathrm{OCH}_{2}\right), 112.5(\mathrm{C}-6$ $\mathrm{Ar}), 121.6$ (C-4 Ar), 123.6 (C-3 Ar), 130.4 (C-3 Ar), 130.6 (C-5 Ar), 132.2 (C-5 Pyrim), 155.9 (C-1 Ar), 156.8 (C-4, C-6 Pyrim), 156.9 (C-2 Pyrim).

Anal. Calcd for $\mathrm{C}_{12} \mathrm{H}_{13} \mathrm{~N}_{3} \mathrm{O}$ : C, 66.96; H, 6.09; N, 19.52. Found: C, 66.92; H, 6.07; N, 19.59.

\section{2-[2-(Thiazol-2-yl)phenoxy]ethanamine (30)}

The title compound was obtained by following General Procedure D from 24 (0.135 g, $0.42 \mathrm{mmol})$ as a dark oil; yield: $0.072 \mathrm{~g}(0.33 \mathrm{mmol}$, $77 \%)$.

IR (neat): 3399 (br), 3233, 1665, 1397, 1099, 1063, 886, $629 \mathrm{~cm}^{-1}$. $\left.{ }^{1} \mathrm{H} \mathrm{NMR} \mathrm{(400} \mathrm{MHz,} \mathrm{CDCl} 3\right): \delta=1.73\left(\right.$ br s, $\left.2 \mathrm{H} \mathrm{NH}_{2}\right), 3.32(\mathrm{t}, J=5.1 \mathrm{~Hz}$, $\left.2 \mathrm{H}, \mathrm{CH}_{2} \mathrm{NH}_{2}\right), 4.28\left(\mathrm{t}, J=5.1 \mathrm{~Hz}, 2 \mathrm{H}, \mathrm{OCH}_{2}\right), 7.07(\mathrm{~d}, J=7.9 \mathrm{~Hz}, 1 \mathrm{H}$, CH-6 Ar), 7.14 (dt, J = 1.0, 7.8 Hz, 1 H, CH-4 Ar), 7.38-7.50 (m, $2 \mathrm{H}, \mathrm{CH}-$ 5 Thia, CH-5 Ar), 7.97 (d, $J=3.6 \mathrm{~Hz}, 1 \mathrm{H}, \mathrm{CH}-4 \mathrm{Thia}$ ), 8.45 (dd, $J=1.6$, $7.8 \mathrm{~Hz}, 1 \mathrm{H}, \mathrm{CH}-3 \mathrm{Ar})$. 
${ }^{13} \mathrm{C} \mathrm{NMR}\left(100 \mathrm{MHz}, \mathrm{CDCl}_{3}\right): \delta=41.7\left(\mathrm{CH}_{2} \mathrm{NH}_{2}\right), 71.5\left(\mathrm{OCH}_{2}\right), 112.0(\mathrm{C}-6$ Ar), 119.5 (C-5 Thia), 121.2 (C-4 Ar), 122.2 (C-2 Ar), 128.6 (C-3 Ar), 130.7 (C-5 Ar), 141.9 (C-4 Thia), 155.4 (C-1 Ar), 162.3 (C-2 Thia).

Anal. Calcd for $\mathrm{C}_{11} \mathrm{H}_{12} \mathrm{~N}_{2} \mathrm{OS}$ : C, 59.97; $\mathrm{H}, 5.49 ; \mathrm{N}, 12.72$. Found: $\mathrm{C}$, 60.13; H, 5.66; N, 12.98 .

\section{2-[2-(Thiazol-5-yl)phenoxy]ethanamine (31)}

The title compound was obtained by following General Procedure D from 25 (0.056 g, $0.17 \mathrm{mmol})$ as a dark oil; yield: $0.030 \mathrm{~g}(0.14 \mathrm{mmol}$, $78 \%)$.

IR (neat): 3283, 3208, 1843, 1666, 1395, 1129, 888, $874 \mathrm{~cm}^{-1}$.

${ }^{1} \mathrm{H} \mathrm{NMR}\left(400 \mathrm{MHz}, \mathrm{CDCl}_{3}\right): \delta=1.58\left(\right.$ br s, $\left.2 \mathrm{H}, \mathrm{NH}_{2}\right), 3.20(\mathrm{t}, J=5.2 \mathrm{~Hz}$, $\left.2 \mathrm{H}, \mathrm{CH}_{2} \mathrm{NH}_{2}\right), 4.14\left(\mathrm{t}, J=5.2 \mathrm{~Hz}, 2 \mathrm{H}, \mathrm{CH}_{2} \mathrm{O}\right), 7.00(\mathrm{~d}, J=8.3 \mathrm{~Hz}, 1 \mathrm{H}$, CH-6 Ar), 7.04 (dt, $J=1.0,7.5 \mathrm{~Hz}, 1 \mathrm{H}, \mathrm{CH}-4 \mathrm{Ar}), 7.27-7.36$ (m, $1 \mathrm{H}, \mathrm{CH}-$ $5 \mathrm{Ar}$ ), 7.67 (dd, $J=1.6,7.5 \mathrm{~Hz}, 1 \mathrm{H}, \mathrm{CH}-3 \mathrm{Ar}$ ), 8.27 (s, $1 \mathrm{H}, \mathrm{CH}-4$ Thia), 8.79 (s, $1 \mathrm{H}, \mathrm{CH}-2$ Thia).

${ }^{13} \mathrm{C}$ NMR $\left(100 \mathrm{MHz}, \mathrm{CDCl}_{3}\right): \delta=41.7\left(\mathrm{CH}_{2} \mathrm{NH}_{2}\right), 71.5\left(\mathrm{OCH}_{2}\right), 112.0(\mathrm{C}-6$ $\mathrm{Ar}), 121.2$ (C-4 Ar), 122.2 (C-2 Ar), 128.6 (C-3 Ar), 130.7 (C-5 Ar), 133.7 (C-5 Thia), 141.1 (C-4 Thia), 153.0 (C-2 Thia), 155.4 (C-1 Ar).

Anal. Calcd for $\mathrm{C}_{11} \mathrm{H}_{12} \mathrm{~N}_{2} \mathrm{OS}$ : C, 59.97; H, 5.49; N, 12.72. Found: $\mathrm{C}$, 60.13; H, 5.66; N, 12.98 .

\section{2-[2-(1-Methyl-1H-pyrazol-4-yl)phenoxy]ethanamine (32)}

The title compound was obtained by following General Procedure D from $26(0.117 \mathrm{~g}, 0.34 \mathrm{mmol})$ as a dark oil; yield: $0.067 \mathrm{~g}(0.31 \mathrm{mmol}$, $83 \%)$.

IR (neat): 3302, 3278, 1782, 1691, 1580, 1385, 113, $858 \mathrm{~cm}^{-1}$.

$\left.{ }^{1} \mathrm{H} \mathrm{NMR} \mathrm{(400} \mathrm{MHz,} \mathrm{CDCl}_{3}\right): \delta=1.55\left(\right.$ br s, $\left.2 \mathrm{H}, \mathrm{NH}_{2}\right), 3.17(\mathrm{t}, J=4.9 \mathrm{~Hz}$, $2 \mathrm{H}, \mathrm{CH}_{2} \mathrm{NH}_{2}$ ), $3.95\left(\mathrm{~s}, 3 \mathrm{H}, \mathrm{NCH}_{3}\right), 4.10\left(\mathrm{t}, J=4.9 \mathrm{~Hz}, 2 \mathrm{H}, \mathrm{OCH}_{2}\right), 6.86$ (d, $J=8.1 \mathrm{~Hz}, 1 \mathrm{H}, \mathrm{CH}-6 \mathrm{Ar}), 6.94(\mathrm{dt}, J=1.2,7.6 \mathrm{~Hz}, 1 \mathrm{H}, \mathrm{CH}-4 \mathrm{Ar}$ ), 7.18-7.27 (m, $1 \mathrm{H}, \mathrm{CH}-5 \mathrm{Ar}), 7.51$ (d, J= 7.6 Hz, $1 \mathrm{H}, \mathrm{CH}-3 \mathrm{Ar}), 7.76-$ 7.90 ( $\mathrm{m}, 2 \mathrm{H}, \mathrm{CH}-4, \mathrm{CH}-5$ Pyra).

${ }^{13} \mathrm{C}$ NMR (100 MHz, CDCl $): \delta=39.1\left(\mathrm{NCH}_{3}\right), 39.7\left(\mathrm{NH}_{2} \mathrm{CH}_{2}\right), 67.4$ $\left(\mathrm{CH}_{2} \mathrm{O}\right.$ ), 112.6 (C-6 Ar), 117.9 (C-4 Pyra), 121.7 (C-2 Ar), 121.7 (C-4 Ar), 127.8 (C-5 Ar), 128.6 (C-3 Ar), 130.1 (C-5 Pyra), 137.0 (C-3 Pyra), $155.2(\mathrm{C}-1 \mathrm{Ar})$.

Anal. Calcd for $\mathrm{C}_{12} \mathrm{H}_{15} \mathrm{~N}_{3} \mathrm{O}$ : C, 66.34; $\mathrm{H}, 6.96 ; \mathrm{N}, 19.34$. Found: C, 66.46; $\mathrm{H}, 7.17$; N, 19.44 .

\section{2-[2-(1-Methyl-1H-imidazol-5-yl)phenoxy]ethanamine (33)}

The title compound was obtained by following General Procedure D from 27 (0.294 g, $0.93 \mathrm{mmol}$ ) as a dark oil; yield: $0.202 \mathrm{~g}(0.92 \mathrm{mmol}$, 99\%).

IR (neat): 3281, 3225, 3045, 1845, 1684, 1394, 1137, 886, $603 \mathrm{~cm}^{-1}$. $\left.{ }^{1} \mathrm{H} \mathrm{NMR} \mathrm{(400} \mathrm{MHz,} \mathrm{CDCl}_{3}\right): \delta=1.37\left(\right.$ br s, $\left.2 \mathrm{H}, \mathrm{NH}_{2}\right), 2.98(\mathrm{t}, J=5.3 \mathrm{~Hz}$, $\left.2 \mathrm{H}, \mathrm{CH}_{2} \mathrm{NH}_{2}\right), 3.55\left(\mathrm{~s}, 3 \mathrm{H}, \mathrm{NCH}_{3}\right), 4.00\left(\mathrm{t}, J=5.3 \mathrm{~Hz}, 2 \mathrm{H}, \mathrm{OCH}_{2}\right), 6.95-$ 7.18 (m, 3 H, CH-4 and CH-6 Ar, CH-4 Imi), 7.21-7.32 (m, $1 \mathrm{H}, \mathrm{CH}-5$ Ar), 7.37-7.47 (m, 1 H, CH-3 Ar), 7.53 (s, 1 H, CH-2 Imi).

${ }^{13} \mathrm{C}$ NMR $\left(100 \mathrm{MHz}, \mathrm{CDCl}_{3}\right): \delta=32.2\left(\mathrm{NCH}_{3}\right), 41.4\left(\mathrm{NH}_{2} \mathrm{CH}_{2}\right), 70.9$ $\left(\mathrm{CH}_{2} \mathrm{O}\right), 112.7$ (C-6 Ar), 119.4 (C-2 Ar), 121.1 (C-4 Ar), 128.7 (C-4 Imi), 129.7 (C-5 Imi), 130.2 (C-5 Ar), 132.2 (C-3 Ar), 138.3 (C-2 Imi), 156.7 (C-1 Ar).

Anal. Calcd for $\mathrm{C}_{12} \mathrm{H}_{15} \mathrm{~N}_{3} \mathrm{O}$ : C, 66.34; $\mathrm{H}, 6.96 ; \mathrm{N}, 19.34$. Found: C, 66.31; $\mathrm{H}, 7.04 ; \mathrm{N}, 19.51$.

\section{References}

(1) Walter, M. W.; Hoffman, B. J.; Gordon, K.; Johnson, K.; Love, P.; Jones, M.; Man, T.; Phebus, L.; Reel, J. K.; Rudyk, H. C.; Shannon, H.; Svensson, K.; Yu, H.; Valli, M. J.; Porter, W. J. Bioorg. Med. Chem. Lett. 2007, 17, 5233.

(2) Elworthy, T.; Ford, A. P.; Bantle, G. W.; Morgans, D. J.; Ozer, R. S.; Palmer, W. S.; Repke, D. B.; Romero, M.; Sandoval, L.; Sjogren, E. B.; Talamás, F. X.; Vazquez, A.; Wu, H.; Arredondo, N. F.; Blue, D. R.; DeSousa, A.; Gross, L. M.; Kava, M. S.; Lesnick, J. D.; Vimont, R. L.; Williams, T. J.; Zhu, Q. M.; Pfister, J. R.; Clarke, D. E. J. Med. Chem. 1997, 40, 2674.

(3) Coban, G.; Zencir, S.; Zupkó, I.; Réthy, B.; Gunes, H. S.; Topcu, Z. Eur. J. Med. Chem. 2009, 44, 2280.

(4) Brenner, M.; Wienrich, M.; Weiser, T.; Bechtel, W-D.; Palluk, R. Ger. Offen DE 19816880 A1, 1999; Chem. Abstr. 1999, ??: 684267

(5) Adams, J. W.; Ramirez, J.; Shi, Y.; Thomsen, W.; Frazer, J.; Morgan, M.; Edwards, J. E.; Chen, W.; Teegarden, B. R.; Xiong, Y.; Al-Shamma, H.; Behan, D. P.; Connolly, D. T. J. Pharmacol. Exp. Ther. 2009, 331, 96.

(6) Xiong, Y.; Teegarden, B. R.; Choi, J. S.; Strah-Pleynet, S.; Decaire, M.; Jayakumar, H.; Dosa, P. I.; Casper, M. D.; Pham, L.; Feichtinger, K.; Ullman, B.; Adams, J.; Yuskin, D.; Frazer, J.; Morgan, M.; Sadeque, A.; Chen, W.; Webb, R. R.; Connolly, D. T.; Semple, G.; Al-Shamma, H. J. Med. Chem. 2010, 53, 4412.

(7) Abrahams, S. L.; Hazen, R. J.; Batson, A. G.; Phillips, A. P. J. Pharmacol. Exp. Ther. 1989, 249, 359.

(8) Wojciechowski, F.; Hudson, R. H. E. J. Am. Chem. Soc. 2008, 130, 12574.

(9) Dikshit, D. K.; Singh, S.; Singh, M. M.; Kamboj, V. P. Indian J. Chem., Sect. B: Org. Chem. Incl. Med. Chem. 1990, 29, 954.

(10) (a) Diederich, F.; Stang, P. J. Appl. Organomet. Chem. 1998, 12, 877. (b) Topics in Current Chemistry; Vol. 219; Miyaura, N., Ed.; Springer: Berlin, 2002.

(11) Hassan, J.; Sevignon, M.; Gozzi, C.; Schulz, E.; Lemaire, M. Chem. Rev. 2002, 102, 1359.

(12) (a) Miyaura, N.; Suzuki, A. Chem. Rev. 1995, 95, 2457. (b) Suzuki, A. J. Organomet. Chem. 1999, 576, 147. (c) Kotha, S.; Lahiri, K.; Kashinath, D. Tetrahedron 2002, 58, 9633. (d) Bellina, F.; Carpita, A.; Rossi, R. Synthesis 2004, 2419.

(13) Hadden, M.; Goodman, A.; Guo, C.; Guzzo, P. R.; Henderson, A. J.; Pattamana, K.; Ruenz, M.; Sargent, B. J.; Swenson, B.; Yet, L.; Liu, J.; He, S.; Sebhat, I. K.; Lin, L. S.; Tamvakopoulos, C.; Qianping Peng, Q.; Kan, Y.; Palyha, O.; Kelly, T. M.; Xiao-Ming Guan, X. M.; Metzger, J. M.; Reitman, M. L.; Ravi, P.; Nargund, R. P. Bioorg. Med. Chem. Lett. 2010, 20, 2912.

(14) Saikia, B.; Boruah, P. R.; Ali, A. A.; Sarma, D. Tetrahedron Lett. 2015, 56, 633.

(15) (a) Kinzel, T.; Zhang, Y.; Buchwald, S. L. J. Am. Chem. Soc. 2010, 132, 14073. (b) Nagaki, A.; Takabayashi, N.; Moriwaki, Y.; Yoshida, J. Chem. Eur. J. 2012, 18, 11871. (c) Molander, G. A.; Canturk, B.; Kennedy, L. E. J. Org. Chem. 2009, 74, 973; and references cited therein.

(16) Deng, J. Z.; Paone, D. V.; Ginnetti, A. T.; Kurihara, H.; Dreher, S. D.; Weissman, S. A.; Stauffer, S. R.; Burgey, C. S. Org. Lett. 2009, 11,345 .

(17) Baudoin, O.; Guénard, D.; Guéritte, F. J. Org. Chem. 2000, 65, 9268. 
Synthesis

L. I. Manasieva et al.

(18) (a) Wolfe, J. P.; Buchwald, S. L. Angew. Chem. Int. Ed. 1999, 38, 2413. (b) Wolfe, J. P.; Singer, R. A.; Yang, B. H.; Buchwald, S. L. J. Am. Chem. Soc. 1999, 121, 9550. (c) Littke, A. F.; Fu, G. C. Angew. Chem. Int. Ed. 1998, 37, 3387. (d) Littke, A. F.; Dai, C.; Fu,

(19) Mori, R.; Kato, A.; Komenoi, K.; Kurasaki, H.; Iijima, T.; Kawagoshi, M.; Kiran, Y. B.; Takeda, S.; Sakai, N.; Konakahara, T. Eur. J. Med. Chem. 2014, 82, 16. 